\title{
PREDICCIÓN DE VOLATILIDAD DE LA RENTABILIDAD DIARIA DEL MERCADO DEL AZÚCAR Y SU APLICACIÓN EN LA RAZÓN DE COBERTURA*
}

Recibido: 15 de junio de 2014 • Aprobado: 10 de noviembre de 2015

\author{
Arody Ortiz Alvarado** \\ Luis Eduardo Girón ${ }^{* * *}$
}

\section{RESUMEN}

Este trabajo predice la volatilidad de la rentabilidad diaria del precio del azúcar, en el período comprendido entre 1 de junio de 2011 y el 24 de octubre de 2013. Los datos diarios utilizados fueron los precios del azúcar, del etanol y la tasa de cambio de la moneda de Brasil (Real) en dólares. Se usaron modelos multivariados de volatilidad autoregresiva condicional generalizada. A partir de la predicción de los precios del azúcar se calcula la razón de cobertura de mínima varianza. Los resultados muestran, que la razón de cobertura es 0.37 , esto significa que, si un productor adverso al riesgo, que tiene la intención de eliminar un porcentaje de la volatilidad de la rentabilidad diaria del mercado mundial del azúcar, y espera vender 25 contratos de azúcar, cada uno de ellos de 50,84 toneladas (1.271 toneladas), el número de contratos optimo tomando cobertura a futuro será 9 y el número de contratos sin tomar cobertura (de contado) será 16.

\section{PALABRAS CLAVE}

Precio de los commodities, precio de la energía, razón de cobertura, series de tiempo no lineales, azúcar.

\section{CLASIFICACIÓN JEL}

G17, G14, Q11, Q41.

\section{CONTENIDO}

Introducción; 1. Datos y procesamiento; 2. Metodología; 3. Marco teórico; 4. Análisis de resultados; 5. Conclusiones y recomendaciones; Bibliografía.

\footnotetext{
Artículo derivado del proyecto de investigación "Predicción de la volatilidad de la rentabilidad diaria del mercado de la azúcar, de acuerdo con el precio internacional, a partir de un modelo de Volatilidad Autoregresiva Condicional Generalizada Multivariado para su aplicación en la razón de cobertura", desarrollado al interior del grupo de investigación en Desarrollo Regional GIDR, (categoría B) del Departamento de Economía, Pontificia Universidad de Cali, y apoyado por el Departamento de Economía. Período de ejecución. Abril de 2013 - Noviembre de 2013.

** Ingeniero Industrial, Universidad del Valle, Cali, Colombia, Especialista en Finanzas, Pontificia Universidad Javeriana, Cali, Colombia. Especialista en Gerencia Tributaria, Universidad ICESI, Cali, Colombia, Magister en Economía, Pontificia Universidad Javeriana, Bogotá, Colombia. Director Financiero y Administrativo, Fundacion Sarmiento Palau (noviembre de 2014 hasta diciembre de 2015). Director Financiero, Ingenio Sancarlos S.A., Tuluá (mayo de 2005 hasta noviembre de 2014), Colombia. Dirección en Cali: Av.10N \# 54-16 Colinas del Bosque. Teléfono: + 57443934 36. Correo electrónico: arodyo@yahoo.com.mx

*** Estadístico profesional, Universidad del Valle, Cali, Colombia. Magister en Economía Aplicada, Universidad del Valle, Cali, Colombia. Diploma en Estudios Avanzados (DEA), en Métodos Matemáticos Aplicados a la Economía y la Empresa, Universidad Nacional de Educación a Distancia (UNED), Madrid, España. PhD. en Economía. Doctorado Interuniversitario DEciDE, Madrid, España. Docente investigador, grupo GIDR, Departamento de Economía, Pontificia Universidad Javeriana Cali, calle 18 No. 118-250, Cali, Colombia. Teléfono + 5743218200 ext. 8341. Correo electrónico: legiron@javerianacali.edu.co.
} 


\section{DAILY PROFITABILITY VOLATILITY FORECAST FOR THE SUGAR MARKET AND ITS APPLICATION FOR THE COVERAGE RATIO}

\section{ABSTRACT}

This work predicts the volatility of the daily profitability of sugar prices for the period between 1 of June 2001 and 24th of October 2013. The daily data collected covered sugar, ethanol and the Brazilian Real and USD exchange rate information. The models used were multivariate generalized conditional autoregressive volatility. Parting from the sugar price prediction, the coverage ratio for the minimum variance is calculated. Results show that the coverage ratio is 0.37 . This means that in the case of a risk adverse producer that wants to eliminate a percentage of volatility of the world's sugar market daily profitability, and expects to sell 25 sugar contracts, 50,84 tons each, (1,271 tons), the optimal number of contracts with future coverage will be 9 and the number of contracts without coverage (spot price) will be 16 .

\section{KEY WORDS}

Price of commodities, Energy price, coverage ratio, non linear time series, sugar.

\section{JEL CLASSIFICATION}

G17, G14, Q11, Q41.

\section{CONTENT}

Introduction; 1. Data and data processing ; 2. Methodology; 3. Theoretical framework; 4. Result Analysis;

5. Conclusions and recommendations; Bibliography.

\section{PREDIÇÃO DE VOLATILIDADE DA RENTABILIDADE DIÁRIA DO MERCADO DO AÇÚCAR E SUA APLICAÇÃO NA RAZÃO DE COBERTURA \\ RESUMO}

Este trabalho prevê a volatilidade da rentabilidade diária do preço do açúcar, no período compreendido entre o dia $1^{\circ}$ de junho de 2011 e 24 de outubro de 2013. Os dados diários utilizados foram os preços do açúcar, do etanol e a taxa de câmbio da moeda do Brasil (Real) em dólares. Se usaram modelos multivariados de volatilidade autorregressiva condicional generalizada. A partir da predição dos preços do açúcar se calcula a razão de cobertura de mínima variância. Os resultados mostram, que a razão de cobertura é 0.37 , isto significa que, se um produtor adverso ao risco, que tem a intenção de eliminar uma porcentagem da volatilidade da rentabilidade diária do mercado mundial do açúcar, e espera vender 25 contratos de açúcar, cada um deles de 50,84 toneladas (1.271 toneladas), o número de contratos ótimo tomando cobertura a futuro será 9 e o número de contratos sem tomar cobertura (de contado) será 16.

\section{PALAVRAS CHAVE}

Preço dos commodities, preço da energia, razão de cobertura, séries de tempo não lineais, açúcar.

\section{CLASSIFICAÇÃO JEL} G17, G14, Q11, Q41.

\section{CONTEÚDO}

Introdução; 1. Dados e processamento; 2. Metodologia; 3. Marco teórico; 4. Análise de resultados; 5. Conclusões e recomendações; Bibliografia. 


\section{INTRODUCCIÓN}

El acelerado proceso de globalización de la economía mundial ha transformado el escenario competitivo para los productores agrícolas. Al respecto FAO (2012) argumenta que el riesgo de mayor volatilidad en los mercados de alimentos en los próximos años, asociado al cambio climático, al crecimiento de los mercados de biocombustibles, a las fluctuaciones en los tipos de cambio y a la entrada de un mayor número de especuladores al mercado, provocará dificultades para acertar el comportamiento de la oferta mundial de bienes agrícolas.

La evidencia reciente en volatilidad de los precios internacionales de los commodities, debido a que están cada vez más expuestos a choques en un mundo con interdependencias económicas, según Arias y otros (2011) hace más compleja la proyección de la producción en términos de costos y precios de realización, generando con ello incertidumbre sobre las economías al producir cambios bruscos e inesperados en la renta de los productores.

De acuerdo con ASOCAÑA (2013), desde el año 2009 el mercado azucarero mundial ha reflejado una alta volatilidad asociada a la implementación de políticas por parte de aquellos países donde se encuentran los más grandes productores (Brasil, India, China, Tailandia) y consumidores de azúcar (EE.UU, India, Unión Europea, China), así como a factores climáticos que afectaron la producción de azúcar de caña y remolacha. La última crisis financiera mundial de 2008 mostró que los precios de los commodities agrícolas estuvieron sujetos a una fuerte volatilidad y los ingenios azucareros en Colombia no han sido ajenos a dichas variaciones.

En un escenario de incertidumbre como el planteado antes, pronosticar la volatilidad de los precios de un commodity como el del azúcar le permite a los ingenios: analizar y manejar con mayor acierto el riesgo financiero, diseñar mejor sus portafolios de inversión, y desarrollar estrategias dinámicas de cobertura. A raíz de esto, diferentes investigaciones se han orientado a analizar la volatilidad de los precios de productos agrícolas como el azúcar. Entre estos se encuentran:

Saban, Cumhur y Ugur (2013), quienes examinan la transmisión de volatilidad entre el petróleo y los precios de los productos básicos agropecuarios (trigo, maíz, soya y azúcar).Para ello, utilizan datos de dos subperíodos, 1986-2005 y 2006-2011, éste último para dar cuenta de la crisis de productos básicos 2006-2008. Los resultados encontrados, entre otros, muestran que no hay transferencia de riesgos entre los mercados de productos agrícolas básicos y el petróleo antes de la crisis. Sin embargo, encuentran evidencia de que, después de la crisis, el riesgo de mercado del petróleo se transmite al maíz, al trigo, y al mercado de soya. Además, descu- 
bren la existencia de spillover ${ }^{1}$ de volatilidad del trigo al mercado del petróleo para ambos períodos; mientras que el mercado del azúcar parece ser neutral al riesgo de mercado del petróleo.

Serra (2011), mediante un modelo GARCH semiparamétrico, estudia los vínculos entre la volatilidad de los precios del petróleo, azúcar y etanol en el período 20002009. Los resultados sugieren fuertes vínculos entre la volatilidad de los precios estudiados.

Gilbert (2010) analiza la tendencia general en la opinión que afirma que la volatilidad de precios de alimentos ha aumentado con el tiempo. La volatilidad se ha incrementado durante los últimos años, pero también ha habido períodos de alta volatilidad en el pasado y, salvo en el caso importante de granos, el reciente episodio no parece excepcional. Destaca en su estudio, que es posible esperar que los niveles de volatilidad se reduzcan a niveles históricos en los próximos años. A través del análisis de causalidad de Granger establece el papel del crecimiento de la demanda, la expansión monetaria y los movimientos del tipo de cambio en la explicación de los movimientos de precios desde 1971. Dentro de las principales conclusiones menciona que la demanda de granos y oleaginosas como materias primas de biocombustible ha sido citada como la principal causa de la subida de precios, pero hay poca evidencia directa para esta afirmación. En cambio, la inversión basada en índices en los mercados de futuros agrícolas es vista como el principal canal a través del cual los factores macroeconómicos y monetarios generan aumentos de los precios de los alimentos en el período 2007-2008.

Riaño (1997) estudia el efecto de los precios de futuros del café, el cacao y el azúcar, sobre la volatilidad del precio spot del café durante el período 1986-1996. A partir del análisis de la función de transferencia y asumiendo que la periodicidad de ocurrencia de las intervenciones se conoce, encuentra que los precios de futuros de los tres productos se forman exógenamente a partir de expectativas sobre el comportamiento de estos productos y del rendimiento que surge por la tenencia de los mismos en el futuro. Otro hallazgo es que aumentos en la variación de las cotizaciones de futuros de la primera, segunda y tercera posición del café producen un aumento en las variaciones del precio spot. En el caso del azúcar no se pudo probar que las posiciones puedan ser consideradas como factores de transferencia de ruido que alteran el comportamiento del precio spot del café durante el período estudiado.

1 Tendencia de cambio en la volatilidad de un activo financiero o mercado, ante un cambio de volatilidad en otro. 
Las investigaciones anteriores no abordan el pronóstico de la volatilidad condicional de la rentabilidad diaria del mercado del azúcar, de acuerdo con el precio internacional, utilizando modelos de volatilidad multivariados, siendo este tipo de estudios escaso. Dada la importancia de mitigar en parte la incertidumbre del productor al planificar sus ingresos, costos, inversión en el cultivo de caña, y su producción de azúcar; esta investigación llena este vacío, pronosticando la volatilidad de la rentabilidad diaria del mercado del azúcar, de acuerdo con el precio spot y precio futuro internacional, a partir de la estimación de la volatilidad en forma simultánea de la rentabilidad del azúcar, el etanol y el tipo de cambio (Dólar por real), utilizando los modelos de Volatilidad Autoregresiva Condicional Generalizada "GARCH" multivariados, para su aplicación en la razón de cobertura para el azúcar.

Dentro de los resultados de la investigación se destacan los siguientes:

- Las series de rendimiento del azúcar, el etanol y el tipo de cambio (Dólar por real), presentan las características típicas de las series financieras, como son: asimétricas y leptocúrticas; presentan ausencia o escasa correlación en las series de rendimientos; poseen varianza cambiante a lo largo del tiempo, alternando períodos de poca volatilidad seguidos de otros de alta volatilidad (agrupamiento de la volatilidad).

- La media no condicionada de los rendimientos de los precios de las tres series analizadas yl (Azúcar), y2 (Etanol), y y3 (Dólar por real) es igual a cero en el largo plazo, es decir, no hay predictibilidad de los retornos de los precios.

- En los momentos de baja volatilidad de los rendimientos del azúcar y baja volatilidad de los rendimientos del etanol, se aprecia una leve correlación y en los momentos de alta volatilidad se aprecia una semifuerte correlación. Esto puede encontrar explicación en que el comportamiento del precio del azúcar posee otros determinantes fundamentales fuertes en el mercado, como son: el stock de inventarios en el mundo como consecuencia de la producción en los diferentes países, el clima que repercute en los rendimientos agrícolas y fabriles, y la participación en las Bolsas de los fondos de especulación que utilizan los commodities como inversión.

- Para el período analizado, la razón de cobertura es 0.37. Esto significa que, si un productor adverso al riesgo espera vender 25 contratos de azúcar, cada uno de ellos de 50,84 toneladas (1.271 toneladas), el número de contratos óptimo con cobertura (de futuro) será aproximadamente de 9. 
Este documento se compone de cinco secciones, adicionales a esta introducción. En la primera, sección se describen los datos y su procesamiento. En la segunda, se explica la metodología seguida para responder los objetivos propuestos. En la tercera, se desarrolla el marco teórico centrado en la teoría de la volatilidad y su modelación. En la cuarta sección, se analizan los resultados de los modelos GARCH multivariados procesados, del pronóstico de la volatilidad de los rendimientos spot y futuros del azúcar y el cálculo de la razón de cobertura de mínima varianza. En la quinta sección, se presentan las conclusiones y recomendaciones para futuras investigaciones.

\section{DATOS Y PROCESAMIENTO}

Los datos ${ }^{2}$ utilizados son: los precios diarios del azúcar publicados en New York Board of Trade (NYBOT), de la posición próxima a expirar para la serie de precios de contado, y la posición de futuro disponible en NYBOT para el mes de mayo del año 2014 para la serie de precios de futuro; los precios diarios del etanol publicados en Brazilian Mercantile and Futures Exchange (BMEF), de la posición próxima a expirar para la serie de precios de contado, y la posición de futuro disponible en BMEF para el mes de marzo de 2014 para la serie de precios de futuro, y la tasa de cambio (dólares por real) diario publicado en New York Board of Trade, al finalizar negociaciones diarias para la serie de precios de contado, y los contratos de futuros para el mes de marzo de 2014 para la serie de precios de futuro, para un período muestral ${ }^{3}$ comprendido desde el 1 de junio de 2011 hasta el 24 de octubre de 2013 para las dos series (precios de contado y precios de futuro). Para el procesamiento de los datos se utiliza la hoja de cálculo Excel y para la estimación de los modelos multivariados se utiliza el software Eviews 8.

\section{METODOLOGÍA}

La metodología seguida para responder al objetivo propuesto en la investigación es:

1. Modelación de la volatilidad multivariada, utilizando las siguientes versiones de los modelos GARCH multivariados: VECH diagonal y BEKK diagonal.

2 Para obtener y consultar los datos que resulten de interés, pueden solicitarse al siguiente correo electrónico: arodyo@yahoo.com.mx

3 Se ha tomado una muestra suficientemente representativa (840 días) de las series de los precios diarios del azúcar, el etanol y el dólar por real con el propósito de que la evaluación o test de eficiencia del mercado del azúcar sea concluyente, es decir, que no haya posibilidad de predicción de los precios por no seguir una tendencia definida, y por tanto se justifique abordar el estudio de la volatilidad y predicción de los rendimientos de los precios diarios de las series mencionadas. 
2. Selección del mejor modelo de volatilidad basado en los criterios estándar de evaluación (Akaike y Schwartz), teniendo en cuenta que la suma de los coeficientes cumplan con el criterio de estacionariedad.

3. A partir del modelo resultante se pronostican 30 períodos adelante de la volatilidad de los retornos de los precios spot y futuros del azúcar, con el fin de obtener la información para el cálculo de la razón de cobertura.

\section{MARCO TEÓRICO}

\subsection{La volatilidad}

De acuerdo con Tsay (2005), el análisis de series de tiempo financieras es una disciplina muy empírica que se refiere a la teoría y práctica de la valoración de los activos en el tiempo. En este contexto la volatilidad que no es observable de un modo directo, se refiere a la desviación o variación de los rendimientos del activo de interés en un período de tiempo ante determinados hechos; la cual es utilizada a menudo como una medida aproximada de riesgo.

La volatilidad de variables financieras se describe por Brooks (2008, p. 383) "como el cálculo de la varianza o desviación estándar de los rendimientos de un activo para un período de tiempo específico. Esta varianza o desviación estándar puede considerarse como el pronóstico de volatilidad para períodos de tiempo en el futuro".

En síntesis, siguiendo a Diebold (2007), la volatilidad es importante para el análisis y manejo de riesgo financiero, selección de portafolio, valoración de activos, y desarrollo de estrategias dinámicas de coberturas; constituyéndose en punto central de la econometría financiera. Si se puede predecir o pronosticar la volatilidad futura de los rendimientos diarios del mercado del azúcar y lograrla introducir en un modelo teórico de valoración, cualquier valor obtenido será mejor que si fuera ignorada la volatilidad.

\subsection{Modelos de volatilidad}

Existen una amplia variedad de modelos de volatilidad. Sin embargo, en este trabajo se consideran solo los modelos univariados y multivariados de volatilidad condicional, enfatizando en los multivariados.

\subsubsection{Modelos univariados de varianza condicional}

Modelos de volatilidad autorregresiva condicional heteroscedástica (ARCH)

Un modelo no lineal en particular, en el uso generalizado de las finanzas, se conoce como un modelo ARCH (Autorregresiva condicional heteroscedástica). La asunción 
de varianza constante del término de error se conoce como homoscedasticidad. Si la varianza de los errores no es constante, esto se conoce como heteroscedasticidad. Es poco probable que en el contexto de series de tiempo financieras la varianza de los errores sea constante en el tiempo y, por lo tanto, tiene sentido considerar un modelo que no asume que la varianza del término de error es constante, y que describa cómo evoluciona la varianza de los errores en un período de tiempo. Otra característica importante de muchas series de rendimientos de los activos financieros que proporciona una motivación para la clase de los modelos $\mathrm{ARCH}$, se conoce como conglomerados de volatilidad (volatility clusters) que describen cómo el nivel actual de la volatilidad, tiende a estar correlacionado de manera positiva con su nivel durante los períodos anteriores o recientes.

El pionero del desarrollo del modelo ARCH fue Engle (1982), quien al relajar el supuesto de independencia estadística permite obtener un modelo lineal que captura la dinámica de la volatilidad condicional, con una estructura particular tal como ARCH. A partir de modelación ARCH es posible modelar la característica de autocorrelación en volatilidad, permitiendo que la varianza condicional sea descrita por una función de los valores pasados del error cuadrático. Entonces denominando a $\sigma_{\mathrm{t}}^{2}$ como $\mathrm{h}_{\mathrm{t}}$, se tiene:

$h_{t}=\alpha_{0}+\alpha_{1} \varepsilon_{t-1}^{2}$

El modelo anterior se conoce como un ARCH (1), ya que la varianza condicional depende de un solo rezago del error cuadrático. Pero se trata sólo de un modelo parcial, ya que nada se ha dicho aún sobre la media condicional. Para incluir la descripción de la dinámica de la media condicional, un ejemplo de un modelo completo sería:

$$
\begin{array}{r}
y_{t}=\beta_{1}+\beta_{2} x_{2 t}+\beta_{3} x_{3 t}+\beta_{4} x_{4 t}+\varepsilon_{t} \operatorname{con} \varepsilon_{t} \sim N\left(0, h_{t}\right) \\
h_{t}=\alpha_{0}+\alpha_{1} \varepsilon_{t-1}^{2}
\end{array}
$$

El modelo anterior podría extenderse al caso general en que la varianza del error $h_{t}$ depende de $q$ rezagos de los errores cuadráticos, que se conoce como un modelo ARCH (q):

$y_{t}=f\left(z_{t}\right)+\varepsilon_{t} \operatorname{con} \varepsilon_{t} \sim N\left(0, h_{t}\right)$

$h_{t}=\alpha_{0}+\sum_{i=1}^{q} \alpha_{i} \varepsilon_{t-i}^{2}$

Donde $\mathrm{f}\left(\mathrm{z}_{\mathrm{t}}\right)$ es una función que modela el valor esperado de $\mathrm{y}_{\mathrm{t}}$. Con, $\alpha_{0}>0, \alpha_{\mathrm{i}} \geq 0, \quad \mathrm{i}=1, \ldots, \mathrm{q}$ y $\sum_{\mathrm{i}=1}^{\mathrm{q}} \alpha_{\mathrm{i}}<1$. 
Hay que señalar que los modelos ARCH (q) rara vez son utilizados porque traen consigo las siguientes limitaciones:

- No resulta fácil decidir el número de rezagos (q), aunque se dispone de una prueba de razón de verosimilitud.

- El orden (q) para capturar la totalidad de la dependencia en la varianza condicional podría resultar muy grande, y esto daría lugar a un modelo poco parsimonioso.

- Los modelos ARCH suponen que los choques positivos y negativos tienen los mismos efectos sobre la volatilidad, ya que esta depende de los choques cuadráticos pasados. En la práctica se reconoce que hay asimetría.

Una extensión natural de un modelo $\mathrm{ARCH}$ (q) que supera algunos de estos problemas es un modelo GARCH. En contraste con el modelo ARCH, el modelo GARCH es muy utilizado en la práctica. De hecho, Bollerslev (1986) propone el modelo GARCH como una ampliación del modelo ARCH.

\section{Modelos de Volatilidad Autoregresiva Condicional Generalizada (GARCH)}

En el modelo $\mathrm{GARCH}$, la volatilidad de $\mathrm{h}_{\mathrm{t}}$ depende de sus propios rezagos, de tal forma que la ecuación de volatilidad en su forma más simple es:

$h_{t}=\alpha_{0}+\alpha_{1} \varepsilon_{t-1}^{2}+\beta \sigma_{t-1}^{2}$

El cual corresponde a un modelo $\operatorname{GARCH}(1,1)$. La interpretación de $\sigma_{t}^{2}$ como $\mathrm{GARCH}$ varianza condicional se debe a que es un estimador de un período hacia adelante de la volatilidad con base en información del pasado relevante. Con base en el modelo, la varianza $h_{t}$ puede ser interpretada como una función ponderada del largo plazo $\left(\alpha_{0}\right)$, más la información del error del período anterior $\left(\alpha_{1} \varepsilon_{\mathrm{t}-1}^{2}\right)$, más la varianza estimada del modelo durante el período anterior $\left(\beta \sigma_{t-1}^{2}\right)$.

El modelo GARCH $(1,1)$ se puede extender a un modelo $\mathrm{GARCH}(\mathrm{q}, \mathrm{p})$, donde la varianza condicional actual se parametriza para depender de q errores al cuadrado y p rezagos de la varianza condicional.

$$
\begin{aligned}
& h_{t}=\alpha_{0}+\alpha_{1} \varepsilon_{t-1}^{2}+\alpha_{2} \varepsilon_{t-2}^{2}+\ldots+\alpha_{q} \varepsilon_{t-q}^{2}+\beta \sigma_{t-1}^{2}+\beta_{2} \sigma_{t-2}^{2}+\beta_{p} \sigma_{t-p}^{2} \\
& h_{t}=\alpha_{0}+\sum_{i=1}^{q} \alpha_{i} \varepsilon_{t-i}^{2}+\sum_{j=1}^{p} \beta_{j} h_{t-j}
\end{aligned}
$$

Donde, $\alpha_{0}>0, \beta_{\mathrm{i}} \geq 0, \beta_{\mathrm{i}} \geq 0, \mathrm{i}=1, \ldots, \mathrm{q}, \mathrm{j}=1, \ldots, \mathrm{p}$ lo que garantiza no incumplir la condición de no negatividad de la varianza condicional. En general, un modelo 
GARCH $(1,1)$ será suficiente para capturar las características de la volatilidad en las series de rendimientos financieros. La varianza condicional está cambiando, pero la varianza no condicional de $\varepsilon_{\mathrm{t}}$ será constante, y dada por:

$$
\mathrm{V}\left(\varepsilon_{\mathrm{t}}\right)=\frac{\alpha_{0}}{1-\sum_{i=1}^{q} \alpha_{i}-\sum_{j=1}^{p} \beta_{j}}
$$

Siempre y cuando se cumpla que $\sum_{\mathrm{i}=1}^{\mathrm{q}} \alpha_{\mathrm{i}}+\sum_{\mathrm{i}=1}^{\mathrm{p}} \beta_{\mathrm{i}}<1$.

Para $\sum_{\mathrm{i}=1}^{\mathrm{q}} \alpha_{\mathrm{i}}+\sum_{\mathrm{i}=1}^{\mathrm{p}} \beta_{\mathrm{i}} \geq 1$ se tiene que la varianza no condicional de $\varepsilon_{\mathrm{t}}$ no se encuentra definida, lo que se denomina no estacionariedad en la varianza; en tal caso, un choque de volatilidad persiste en el tiempo. Cuando $\sum_{\mathrm{i}=1}^{\mathrm{q}} \alpha_{\mathrm{i}}+\sum_{\mathrm{i}=1}^{\mathrm{p}} \beta_{\mathrm{i}}=1$ es conocido como "raíz unitaria en la varianza", también denominado "Integrado GARCH "o IGARCH . La no-estacionariedad de la varianza no tiene mucha motivación teórica, como sería el caso para la no estacionariedad en la media (observada en la serie de precios). Así, un GARCH cuyos coeficientes tiendan a adoptar valores que conlleven a una varianza no condicional no estacionaria presentan propiedades no deseables, tales como problemas de convergencia a la varianza de largo plazo cuando se lleva a cabo un pronóstico de esta.

Vale la pena señalar que una de las debilidades entre otras de los modelos GARCH es la dificultad de capturar por completo los efectos Leverage, entendidos como la reacción de la volatilidad a caídas o subidas del precio de la misma magnitud, pues el modelo GARCH asume que dicha reacción es simétrica, lo cual no sucede en la realidad, pues la volatilidad condicional reacciona más fuertemente ante una caída que ante una subida del precio en la misma magnitud. No obstante esta debilidad el GARCH captura el fenómeno de Volatility Clusters, es decir, agrupamiento de la volatilidad en períodos de alta volatilidad y períodos de baja volatilidad.

Aunque los modelos univariados capturan las características estilizadas de la volatilidad, tienen una limitación por no considerar los movimientos conjuntos de volatilidad de varios mercados o activos diferentes; es decir, no es posible modelar las varianzas y su correspondiente correlación en forma simultánea. Por consiguiente, los modelos GARCH multivariados contribuyen en forma para solucionar dicha debilidad y para solucionar las dificultades de los modelos univariados. 


\subsubsection{Modelos multivariados de varianza condicional}

Bollerslev, Engle y Wooldridge (1988) amplían la metodología de los modelos univariados al contexto multivariado, relajando el supuesto homoscedasticidad para las covarianzas. Los modelos GARCH multivariados son de la misma naturaleza que los modelos GARCH univariados. La diferencia consiste en que los modelos multivariados tienen, además, ecuaciones para las covarianzas cambiantes en el tiempo, consiguiendo simplificar las relaciones dinámicas entre los procesos de volatilidad de los rendimientos de varios activos. En este sentido, un contexto multivariado con matriz de varianzas y covarianzas condicional de los rendimientos no constante es el marco econométrico apropiado para el análisis que permita capturar el cambio de tendencia en la volatilidad de la rentabilidad del mercado del azúcar, ante cambios en la volatilidad de la rentabilidad del mercado del etanol, así como cambios en la volatilidad de la rentabilidad de la tasa de cambio de Brasil (Dólar por real).

Varias formulaciones diferentes a los modelos GARCH multivariantes se han propuesto en la literatura, incluyendo el modelo VECH, el modelo VECH diagonal, el modelo BKK, el modelo BKK diagonal, y el modelo de correlación condicional constante (CCC). A continuación se describe cada uno de los anteriores modelos.

\section{El modelo VECH}

Siguiendo a Brooks (2008), la siguiente es la especificación inicial propuesta por Bollerslev, Engle y Wooldridge (1988) para la representación de un modelo GARCH multivariado:

$\operatorname{Vech}\left(\mathrm{H}_{t}\right)=\mathrm{C}+\sum_{i=1}^{q} \mathrm{~A}_{i} \operatorname{Vech}\left(\varepsilon_{t-i} \varepsilon_{t-i}\right)+\sum_{j=1}^{p} \mathrm{~B}_{j} \operatorname{Vech}\left(\mathrm{H}_{t-i}\right), \operatorname{con} \varepsilon_{\mathrm{t}} \mid \varphi_{\mathrm{t}} \sim \mathrm{N}\left(0, \mathrm{H}_{\mathrm{t}}\right)$

También se puede representar a partir de una forma vectorial, expresada de la siguiente manera:

$$
\operatorname{VECH}\left(\mathrm{H}_{\mathrm{t}}\right)=\mathrm{C}+\operatorname{AVECH}\left(\Xi_{\mathrm{t}-1} \Xi_{\mathrm{t}-1}^{\prime}\right)+\operatorname{BVECH}\left(\mathrm{H}_{\mathrm{t}-1}\right) \operatorname{con} \Xi_{\mathrm{t}} \mid \Psi_{\mathrm{t}-1} \sim \mathrm{N}\left(0, \mathrm{H}_{\mathrm{t}}\right)
$$

donde $H_{t}$ es la matriz de covarianzas condicionales, $\Xi_{t}$ es el vector de perturbaciones, $\Psi_{t-1}$ representa el conjunto de información disponible en t, C es un vector de interceptos, A y B son matrices de parámetros y $\mathrm{VECH}($.$) es el operador$ que vectoriza la parte triangular superior de la matriz $\left(\Xi_{\mathrm{t}} \Xi_{\mathrm{t}}\right)$ simétrica $\mathrm{N} x \mathrm{~N}$ en un vector de dimensión $\mathrm{N}^{*}=(\mathrm{N}(\mathrm{N}+1) / 2)$.

En el caso bivariado se tiene: 


$$
\mathrm{H}_{t}=\left[\begin{array}{ll}
h_{11 t} & h_{12 t} \\
h_{21 t} & h_{22 t}
\end{array}\right], \Xi_{t}=\left[\begin{array}{l}
\varepsilon_{1 t} \\
\varepsilon_{2 t}
\end{array}\right], \mathrm{C}=\left[\begin{array}{l}
\omega_{11} \\
\omega_{21} \\
\omega_{31}
\end{array}\right], \mathrm{A}=\left[\begin{array}{lll}
\alpha_{11} & \alpha_{12} & \alpha_{13} \\
\alpha_{21} & \alpha_{22} & \alpha_{23} \\
\alpha_{31} & \alpha_{32} & \alpha_{33}
\end{array}\right], \mathrm{B}=\left[\begin{array}{lll}
\beta_{11} & \beta_{12} & \beta_{13} \\
\beta_{21} & \beta_{22} & \beta_{23} \\
\beta_{31} & \beta_{32} & \beta_{33}
\end{array}\right]
$$

El modelo bivariado requiere la estimación de 21 parámetros ( $\mathrm{C}$ tiene 3 elementos; A y B, tienen 9 elementos cada uno). Los elementos del modelo VECH se escriben a continuación.

El operador VECH(.) se aplica a la triangular superior de la matriz simétrica, generando un vector con una sola columna. En el caso $\operatorname{VECH}\left(\mathrm{H}_{\mathrm{t}}\right)$, esto se convierte en:

$$
\operatorname{VECH}\left(\mathrm{H}_{t}\right)=\left[\begin{array}{l}
h_{11 t} \\
h_{22 t} \\
h_{12 t}
\end{array}\right]
$$

En el caso de $\operatorname{VECH}\left(\Xi_{\mathrm{t}-1} \Xi_{\mathrm{t}-1}^{\prime}\right)$ puede ser expresado como:

$$
\begin{gathered}
\operatorname{VECH}\left(\Xi_{\mathrm{t}} \Xi_{\mathrm{t}}^{\prime}\right)=\left[\begin{array}{c}
\varepsilon_{1 t-1}^{2} \\
\varepsilon_{2 t-1}^{2} \\
\varepsilon_{1 t-1} \varepsilon_{2 t-1}
\end{array}\right] \\
\operatorname{VECH}\left(\mathrm{H}_{t-1}\right)=\left[\begin{array}{l}
h_{11 t-1} \\
h_{22 t-1} \\
h_{12 t-1}
\end{array}\right]
\end{gathered}
$$

El modelo VECH completo está representado por las siguientes ecuaciones:

$$
\begin{array}{r}
h_{11 t}=\omega_{11}+\alpha_{11} \varepsilon_{2 t-1}^{2}+\alpha_{12} \varepsilon_{2 t-1}^{2}+\alpha_{13} \varepsilon_{1 t-1} \varepsilon_{2 t-1}+\beta_{11} h_{11 t-1}+\beta_{12} h_{22 t-1} \\
h_{11 t}=\omega_{11}+\alpha_{11} \varepsilon_{2 t-1}^{2}+\alpha_{12} \varepsilon_{2 t-1}^{2}+\alpha_{13} \varepsilon_{1 t-1} \varepsilon_{2 t-1}+\beta_{11} h_{11 t-1}+\beta_{12} h_{22 t-1}+\beta_{13} h_{12 t-1} \\
h_{22 t}=\omega_{21}+\alpha_{21} \varepsilon_{1 t-1}^{2}+\alpha_{22} \varepsilon_{2 t-1}^{2}+\alpha_{23} \varepsilon_{1 t-1} \varepsilon_{2 t-1}+\beta_{21} h_{11 t-1}+\beta_{22} h_{22 t-1}+\beta_{23} h_{12 t-1} \\
h_{12 t}=\omega_{31}+\alpha_{31} \varepsilon_{1 t-1}^{2}+\alpha_{32} \varepsilon_{2 t-1}^{2}+\alpha_{33} \varepsilon_{1 t-1} \varepsilon_{2 t-1}+\beta_{31} h_{11 t-1}+\beta_{32} h_{22 t-1}+\beta_{33} h_{12 t-1}
\end{array}
$$

Por lo tanto, es evidente que las varianzas y covarianzas condicionales dependen de los valores rezagados de todas las varianzas condicionales y co- 
varianzas condicionales entre todos los rendimientos de los activos en la serie, así como de los errores al cuadrado rezagados y también de los productos cruzados de los errores cuadráticos. Incluso para solo dos activos, es un trabajo de estimación arduo.

\section{El modelo VECH diagonal}

Incluso en el caso bivariado, la varianza condicional y las ecuaciones de covarianza para el modelo VECH sin restricciones contienen 21 parámetros. En la medida que el número de activos a emplear en el modelo crezca, la estimación del modelo VECH se vuelve menos parsimoniosa y puede convertirse bastante rápido en inviable. Por lo tanto, en la varianza condicional del modelo VECH, la matriz de covarianza se ha restringido, suponiendo que las matrices A y B son diagonales. Esto reduce a 9 el número de parámetros a estimar (ahora las matrices A y B tienen 3 elementos, cada una). El resultado es un modelo más simple, conocido como un VECH diagonal, sin interdependencia dinámica cruzada entre las series de volatilidades y, a pesar de que permite reducir el número de parámetros desconocidos, no garantiza que la matriz de varianza y covarianza sea definida de manera positiva en cada período. Su especificación es la siguiente:

$$
h_{i, t}=\omega_{i j}+\alpha_{i j} \varepsilon_{i, t-1} \varepsilon_{j, t-1}+\beta_{i j} h_{i, t, t-1} \text { para } i, j=1,2
$$

Para el caso bivariado se tiene:

$$
\left[\begin{array}{ll}
h_{11, t} & \\
h_{21, t} & h_{22, t}
\end{array}\right]=\left[\begin{array}{ll}
\omega_{11} & \\
\omega_{12} & \omega_{22}
\end{array}\right]+\left[\begin{array}{ll}
\alpha_{11} & \\
\alpha_{12} & \alpha_{22}
\end{array}\right] *\left[\begin{array}{cc}
\varepsilon_{1, t-1}^{2} & \\
\varepsilon_{1, t-1} \varepsilon_{2, t-1} & \varepsilon_{2, t-1}^{2}
\end{array}\right]+\left[\begin{array}{ll}
\beta_{11} & \\
\beta_{12} & \beta_{22}
\end{array}\right] *\left[\begin{array}{ll}
h_{11, t-1} & \\
h_{21, t-1} & h_{22, t-1}
\end{array}\right]
$$

El modelo VECHdiagonal está representado por las siguientes ecuaciones:

$$
\begin{aligned}
& h_{1 t}=\omega_{11}+\alpha_{11} \varepsilon_{1, t-1}^{2}+\beta_{11} h_{1, t-1} \\
& h_{12, t}=\omega_{12}+\alpha_{12} \varepsilon_{1, t-1} \varepsilon_{2, t-1}+\beta_{12} h_{12, t-1} \\
& h_{2, t}=\omega_{22}+\alpha_{22} \varepsilon_{2, t-1}^{2}+\beta_{22} h_{2, t-1}
\end{aligned}
$$

\section{Modelo BEKK}

El modelo BEKK propuesto y ajustado por Engle y Kroner (1995), permite solucionar la dificultad del modelo VECH de garantizar que la matriz $\mathrm{H}_{\mathrm{t}}$ de varianzas y covarianzas sea definida positiva en cada período, y permite capturar la dependencia dinámica 
entre las series de las volatilidades. Es decir, permite una dependencia directa entre la varianza condicional de una variable, con respecto a los datos observados de las varianzas condicionales de otras variables. Su representación es:

$$
\mathrm{H}_{t}=\mathrm{C}^{\prime} \mathrm{C}+\sum_{i=1}^{q} \mathrm{~A}_{i}\left(\varepsilon_{t-i} \varepsilon_{t-i}^{\prime}\right) \mathrm{A}_{i}^{\prime}+\sum_{j=1}^{p} \mathrm{~B}_{j}\left(\mathrm{H}_{t-j}\right) \mathrm{B}_{j}^{\prime}
$$

También se puede representar así:

$$
\mathrm{H}_{t}=\mathrm{C}^{\prime} \mathrm{C}+\mathrm{A}^{\prime}\left(\Xi_{t-1} \Xi_{t-1}^{\prime}\right) \mathrm{A}+\mathrm{B}^{\prime}\left(\mathrm{H}_{t-1}\right) \mathrm{B}
$$

Donde $C$ es una matriz triangular inferior de constantes y $A_{i}$ y $B_{j}$ son matrices de parámetros. La dimensión de $C, A_{i}$ y $B_{j}$ es kxk, siendo k el número de variables. Los parámetros fuera de la diagonal de las matrices $\mathrm{A}_{i}$ y $\mathrm{B}_{\mathrm{i}}$ recogen los efectos cruzados de mercado en la volatilidad.

En el caso bivariado, el modelo es:

$$
\begin{aligned}
{\left[\begin{array}{ll}
h_{1, t} & \\
h_{12, t} & h_{2, t}
\end{array}\right] } & =\left[\begin{array}{ll}
\omega_{11} & \\
\omega_{12} & \omega_{22}
\end{array}\right] \times\left[\begin{array}{ll}
\omega_{11} & \omega_{12} \\
& \omega_{22}
\end{array}\right]+\left[\begin{array}{ll}
\alpha_{11} & \alpha_{21} \\
\alpha_{12} & \alpha_{22}
\end{array}\right] \times\left[\begin{array}{cc}
\varepsilon_{1, t-1}^{2} & \varepsilon_{1, t-1} \varepsilon_{2, t-1} \\
\varepsilon_{1, t-1} \varepsilon_{2, t-1} & \varepsilon_{2, t-1}^{2}
\end{array}\right] \times\left[\begin{array}{ll}
\alpha_{11} & \alpha_{12} \\
\alpha_{21} & \alpha_{22}
\end{array}\right] \\
& +\left[\begin{array}{ll}
\beta_{11} & \beta_{21} \\
\beta_{12} & \beta_{22}
\end{array}\right] \times\left[\begin{array}{cc}
h_{1, t} & \\
h_{12, t-1} & h_{2, t}
\end{array}\right] \times\left[\begin{array}{ll}
\beta_{11} & \beta_{12} \\
\beta_{21} & \beta_{22}
\end{array}\right]
\end{aligned}
$$

\section{Modelo BEKK diagonal}

Siguiendo a Engle y Kroner (1995), el modelo BEKK diagonal es una representación ajustada del modelo BEKK para lograr una estimación relativamente simple, cuando las matrices $A_{i}$, $B_{i}$ tienen estructura diagonal, se prescinde de las interacciones entre volatilidades. Es un caso particular del modelo VECH diagonal. En el caso bivariado el modelo BEKK diagonal tendría 7 parámetros, frente a los 9 parámetros del modelo VECH diagonal.

$$
\begin{aligned}
{\left[\begin{array}{ll}
h_{1, t} & \\
h_{12, t} & h_{2, t}
\end{array}\right] } & =\left[\begin{array}{ll}
\omega_{11} & \\
\omega_{12} & \omega_{22}
\end{array}\right] \times\left[\begin{array}{ll}
\omega_{11} & \omega_{12} \\
& \omega_{22}
\end{array}\right]+\left[\begin{array}{ll}
\alpha_{11} & \\
& \alpha_{22}
\end{array}\right] \times\left[\begin{array}{cc}
\varepsilon_{1, t-1}^{2} & \varepsilon_{1, t-1} \varepsilon_{2, t-1} \\
\varepsilon_{1, t-1} \varepsilon_{2, t-1} & \varepsilon_{2, t-1}^{2}
\end{array}\right] \times\left[\begin{array}{ll}
\alpha_{11} & \\
& \alpha_{22}
\end{array}\right] \\
& +\left[\begin{array}{ll}
\beta_{11} & \\
& \beta_{22}
\end{array}\right] \times\left[\begin{array}{cc}
h_{1, t} & \\
h_{12, t-1} & h_{2, t}
\end{array}\right] \times\left[\begin{array}{ll}
\beta_{11} & \\
& \beta_{22}
\end{array}\right]
\end{aligned}
$$

No obstante lo anterior, vale señalar que los modelos VECH y BEKK en sus dos versiones presentan dificultades para la interpretación de los parámetros de 
las matrices $A_{i}$ y $B_{j}$, y la modelación de la matriz de covarianza condicional presenta inconvenientes, puesto que la evidencia empírica ha demostrado que la mayoría de los parámetros estimados no son significativos estadísticamente.

Para analizar la volatilidad financiera, con influencias de incertidumbre de la rentabilidad del mercado del azúcar, los modelos estimados en esta investigación, utilizando el software Eviews 8, son los trivariados BEKK diagonal y VECH diagonal. Eviews no estima la forma general de en la que y son de libre disposición.

\section{Modelo CCC}

Con el fin de resolver el problema de dimensionalidad del vector de perturbaciones (), Bollerslev (1990) propone el caso especial en el que, las correlaciones condicionales son invariantes en el tiempo. Este modelo es conocido como condicional de correlación contante (CCC), supone que la matriz de correlaciones condicionales es constante en el tiempo y que las varianzas condicionales son determinadas de acuerdo a modelos GARCH univariados. Las covarianzas condicionales están determinadas por la relación entre la matriz de correlaciones condicionales y la matriz diagonal de desviaciones estándar condicionales.

El modelo de volatilidad se compone de dos ecuaciones para $\Xi_{t}^{*}=\left[\begin{array}{ll}h_{11, t}, & h_{22, t}\end{array}\right]$ y la representación GARCH $(1,1)$ para $\Xi_{t}^{*}=\left[h_{11, t}, \quad h_{22, t}\right]$ es:

$\Xi_{t}^{*}=w_{0}+\alpha_{1} \varepsilon_{t-1}^{2}+\beta_{1} \Xi_{t-1}^{*}$

Donde $\varepsilon_{t-1}^{2}=\left(\varepsilon_{1, t-1}^{2}, \varepsilon_{2, t-1}^{2}\right), w_{0}$ es un vector bidimensional y $\alpha_{1}$ y $\beta_{1}$ son matrices (2x2) no negativas definidas.

En forma ampliada el modelo es:

$\left[\begin{array}{l}h_{11, t} \\ h_{22, t}\end{array}\right]=\left[\begin{array}{l}w_{10} \\ w_{20}\end{array}\right]+\left[\begin{array}{ll}\alpha_{11} & \alpha_{12} \\ \alpha_{21} & \alpha_{22}\end{array}\right]\left[\begin{array}{l}\varepsilon_{1, t-1}^{2} \\ \varepsilon_{2, t-1}^{2}\end{array}\right]+\left[\begin{array}{ll}\beta_{11} & \beta_{12} \\ \beta_{21} & \beta_{22}\end{array}\right]\left[\begin{array}{l}h_{11, t-1} \\ h_{22, t-1}\end{array}\right], \alpha_{i 0}>0$

Tabla 1. Resumen diferentes modelos descritos

\begin{tabular}{|c|c|c|}
\hline Modelo & Representación & \multicolumn{1}{c|}{ Característica } \\
\hline VECH & $\operatorname{Vech}\left(H_{t}\right)=C+\sum_{i=1}^{q} A_{i} \operatorname{Vech}\left(\varepsilon_{t-i} \varepsilon_{t-i}^{\prime}\right)+\sum_{j=1}^{p} B_{j} \operatorname{Vech}\left(H_{t-j}\right)$, & $\begin{array}{l}\text { El VECH completo permite } \\
\text { una interacción entre las } \\
\text { volatilidades de todas las } \\
\text { variables, a costa de un el } \\
\text { evado número de parámet- } \\
\text { ros. }\end{array}$ \\
\hline
\end{tabular}




\begin{tabular}{|c|c|c|}
\hline Modelo & Representación & Característica \\
\hline $\begin{array}{l}\text { VECH } \\
\text { Diagonal }\end{array}$ & $h_{i j, t}=\omega_{i j}+\alpha_{i j} \varepsilon_{i, t-1} \varepsilon_{j, t-1}+\beta_{i j} h_{i j, t-1}$ para $i, j=1,2$ & $\begin{array}{l}\text { En la varianza condicional } \\
\text { del modelo VECH, la matriz } \\
\text { de covarianza se ha restrin- } \\
\text { gido, suponiendo que las } \\
\text { matrices A y B son diago- } \\
\text { nales permitiendo reducir } \\
\text { el número de parámetros a } \\
\text { estimar. }\end{array}$ \\
\hline $\begin{array}{l}\text { BEKK y BEKK } \\
\text { Diagonal }\end{array}$ & $\mathrm{H}_{t}=\mathrm{C}^{\prime} \mathrm{C}+\sum_{i=1}^{q} \mathrm{~A}_{i}\left(\varepsilon_{t-i} \varepsilon_{t-i}^{\prime}\right) \mathrm{A}_{i}^{\prime}+\sum_{j=1}^{p} \mathrm{~B}_{j}\left(\mathrm{H}_{t-i}\right) \mathrm{B}_{j}^{\prime}$ & $\begin{array}{l}\text { El BEKK garantiza que la } \\
\text { matriz de varianzas y cova- } \\
\text { rianzas sea positiva definida } \\
\text { en cada período, mediante } \\
\text { una estructura de evolución } \\
\text { temporal. } \\
\text { El BEKK Diagonal es una } \\
\text { versión simplificada del } \\
\text { BEKK que excluye las inte- } \\
\text { racciones entre volatilida- } \\
\text { des y es un caso particular } \\
\text { del VECH Diagonal. }\end{array}$ \\
\hline CCC & $\begin{array}{l}\Xi_{t}^{*}=w_{0}+\alpha_{1} \varepsilon_{t-1}^{2}+\beta_{1} \Xi_{t-1}^{*} \text {, donde } \varepsilon_{t-1}^{2}=\left(\varepsilon_{1, t-1}^{2}, \varepsilon_{2, t-1}^{2}\right), w_{0} \\
\text { es un vector bidimensional y } \alpha_{1} \text { y } \beta_{1} \text { son matrices } \\
\text { (2x2) no negativas definidas. }\end{array}$ & $\begin{array}{l}\text { Este modelo supone que } \\
\text { la matriz de correlaciones } \\
\text { condicionales es constante } \\
\text { en el tiempo y que las va- } \\
\text { rianzas condicionales son } \\
\text { determinadas de acuerdo } \\
\text { a modelos Garch univaria- } \\
\text { dos. }\end{array}$ \\
\hline
\end{tabular}

Fuente: elaboración propia

\section{Estimación GARCH multivariante}

Los parámetros de los modelos GARCH multivariados,, bajo el supuesto de normalidad condicional, se pueden estimar mediante la maximización de la función de log-verosimilitud que se especifica mediante:

$\ell(\theta)=-\frac{\mathrm{TN}}{2} \log 2 \pi-\frac{1}{2} \sum_{t=1}^{\mathrm{T}}\left(\log \mid \mathrm{H}_{t}+\Xi_{t}^{\prime} \mathrm{H}_{t}^{-1} \Xi_{t}\right)$

Donde $\theta$ es un vector que contiene todos los parámetros desconocidos, $\mathrm{N}$ es el número de los activos (es decir, el número de serie en el sistema) y T es el número de observaciones. La estimación de máxima verosimilitud de $\theta$ es asintóticamente normal, y en consecuencia el proceso de inferencia estadística tradicional es aplicable. 


\section{Pronóstico GARCH multivariante}

Siguiendo a Tsay (2005), una vez obtenido el modelo que mejor explica el comportamiento de la volatilidad condicional de la rentabilidad del mercado del azúcar, es posible obtener el pronóstico fuera de la muestra de la volatilidad asociada a los mismos. Dichos pronósticos son necesarios para el cálculo de la razón de cobertura. La predicción de la volatilidad del modelo BEKK se puede obtener mediante el uso de métodos de predicción similares a las de un vector ARMA $(1,1)$.

El pronóstico de un modelo ARCH puede ser obtenido de manera recursiva como el de un modelo AR, considerando un modelo ARCH (m).

El primer paso de la predicción de $\sigma_{\mathrm{h}+1}^{2}$ es:

$\sigma_{h}^{2}(1)=\alpha_{0}+\alpha_{1} \varepsilon_{h}^{2}+\ldots+\alpha_{m} \varepsilon_{h+1-m}^{2}$

El segundo, es:

$\sigma_{h}^{2}(2)=\alpha_{0}+\alpha_{1} \sigma_{h}^{2}(1)+\alpha_{2} \varepsilon_{h}^{2}+\ldots+\alpha_{m} \varepsilon_{h+2-m}^{2}$

y 1 pasos hacia delante, la predicción para $\sigma_{\mathrm{h}+1}^{2}$ es:

$\sigma_{h}^{2}(l)=\alpha_{0}+\sum_{i=1}^{m} \alpha_{i} \sigma_{h}^{2}(l-i)$

Donde $\sigma_{h}^{2}(l-i)=\varepsilon_{h+l-i}^{2}$ if $(l-i) \leq 0$.

Ahora bien, para la predicción fuera de muestra de la volatilidad de los retornos del precio del azúcar del modelo BEKK, el primer paso de la predicción de la volatilidad es:

$\Xi_{h}(1)=\alpha_{0}+\alpha_{1} \varepsilon_{h}^{2}+\beta_{1} \Xi_{h}$

y para el pronóstico de la volatilidad $l$ pasos hacia delante, se tiene:

$\Xi_{h}(l)=\alpha_{0}+\left(\alpha_{1}+\beta_{1}\right) \Xi_{h}(l-1), l>1$

\section{Estimación de razón de cobertura de mínima varianza (Variance Hedge Ratio).}

La Razón de cobertura dinámica constituye una de las medidas más utilizadas como estrategia para afrontar el riesgo. Consiste en tomar posiciones contrarias en el mercado spot y en el mercado forward al mismo tiempo. El ejercicio de hedging, por medio de la razones de cobertura busca compensar un movimiento adverso y sostenido en el precio de un mercado con la dinámica favorable del precio en el otro mercado. El número de unidades de activos a futuro comprados como proporción del número de unidades de activos spot se conoce como Razón de cobertura de varianza mínima (Hedge Ratio). Dado que el riesgo, en este contexto es la volatilidad 
de los retornos del portafolio, la estrategia consiste en elegir dicha razón de manera que permita minimizar la varianza de los retornos del portafolio compuesto por la posición spot y la posición a futuro (Razón óptima de cobertura). El uso de modelos GARCH multivariados facilita la estimación y pronóstico de las desviaciones estándar y correlación entre los movimientos spot y futuros en forma cambiante a través del tiempo. De esta forma, el valor óptimo de la relación de cobertura se puede determinar, de acuerdo con Hull (2009, p. 55), por definición, utilizando la siguiente notación:

$\delta$ S: cambio en el precio al contado $\mathrm{S}$, durante un período de tiempo igual a la duración de la cobertura. $\delta \mathrm{F}$ : Cambio en el precio del futuro, F, durante un período de tiempo igual a la duración de la cobertura. $\sigma_{\mathrm{S}}$ : Desviación estándar de $\delta \mathrm{S} . \sigma_{\mathrm{F}}$ : Desviación estándar de $\delta \mathrm{F} . \quad \rho$ : Coeficiente de correlación entre $\delta \mathrm{S}$ y $\delta \mathrm{F}$. h*: Ratio de cobertura que minimiza la varianza de la posición del coberturista.

La varianza del portafolio puede ser obtenida a partir de $\operatorname{Var}(\Delta S-h \Delta F)$. Recordando las reglas para manipular el operador de varianza, esto puede ser escrito como $\operatorname{Var}(\Delta \mathrm{S})+\operatorname{Var}(\mathrm{h} \Delta \mathrm{F})-2 \operatorname{Cov}(\Delta \mathrm{S}, \mathrm{h} \Delta \mathrm{F}) \quad \mathrm{O} \operatorname{Var}(\Delta \mathrm{S})+\mathrm{h}^{2} \operatorname{Var}(\Delta \mathrm{F})-2 \mathrm{~h} \operatorname{Cov}(\Delta \mathrm{S}, \Delta \mathrm{F})$. Por lo tanto, la varianza del cambio en el valor de la posición cubierta está dada por $\mathrm{v}=\sigma_{\mathrm{S}}^{2}+\mathrm{h}^{2} \sigma_{\mathrm{F}}^{2}-2 \mathrm{~h} \rho \sigma_{\mathrm{S}} \sigma_{\mathrm{F}}$. Esto puede escribirse como $\left(\mathrm{h} \sigma_{\mathrm{F}}-\rho \sigma_{\mathrm{S}}\right)^{2}+\sigma_{\mathrm{S}}^{2}-\rho^{2} \sigma_{\mathrm{S}}^{2}$. El segundo y el tercer término no incluyen $h$. Por tanto, la varianza se minimiza cuando $\left(\mathrm{h} \sigma_{\mathrm{F}}-\rho \sigma_{\mathrm{S}}\right)^{2}$ es cero, es decir, cuando $\mathrm{h}=\rho \frac{\sigma_{\mathrm{S}}}{\sigma_{\mathrm{F}}}$.

De acuerdo con Brooks (2008), con la anterior fórmula, el índice de cobertura óptima es invariante en el tiempo, y se calcula a partir de datos históricos. Sin embargo, las desviaciones estándar y la correlación entre los retornos de los precios contado y futuro están cambiando en el tiempo, y podrían ser pronosticadas a partir de un modelo GARCH multivariante, de modo que la expresión anterior puede ser sustituida por:

$\mathrm{h}_{\mathrm{t}}=\rho_{\mathrm{t}} \frac{\sigma_{\mathrm{s}, \mathrm{t}}}{\sigma_{\mathrm{F}, \mathrm{t}}}$

Donde $h_{t}$ es razón de cobertura cambiante en el tiempo, $\sigma_{s, t}$ es la desviación estándar del cambio en el precio spot durante la cobertura en el momento t, $\sigma_{\mathrm{F}, \mathrm{t}}$ es la desviación estándar del cambio en el precio forward durante la cobertura en el momento t, y $\rho_{\mathrm{t}}$ es la correlación, durante la cobertura, entre el cambio en el precio spot y el cambio en el precio forward en el momento t.

\section{ANÁLISIS DE RESULTADOS}

El presente capítulo inicia con una descripción estadística de los retornos de las series utilizadas. Luego, se analiza el comportamiento de las series estudiadas para validar los hechos estilizados de las series financieras, es decir, distribuciones no normales asimétricas y leptokúrticas, con escasa correlación y varianzas cambiantes. 
Posteriormente, se continúa con la estimación y pronóstico de la volatilidad de la rentabilidad del mercado del azúcar, de acuerdo con el precio internacional, para por último, calcular la razón de cobertura.

\section{Estadísticas descriptivas de la series de rentabilidad diaria}

Para el período muestral comprendido desde el 1 de junio de 2011 hasta el 24 de octubre de 2013, se observa que los rendimientos promedio de, los precios del azúcar (Y1), y el dólar por real (Y3) negociados en NYBOT, y los precios del etanol (Y2) negociado en BMEF, es apenas superior a cero, por lo que en promedio no hay ni pérdidas ni ganancias significativas.

\section{Gráfica 1. Series de rendimientos}

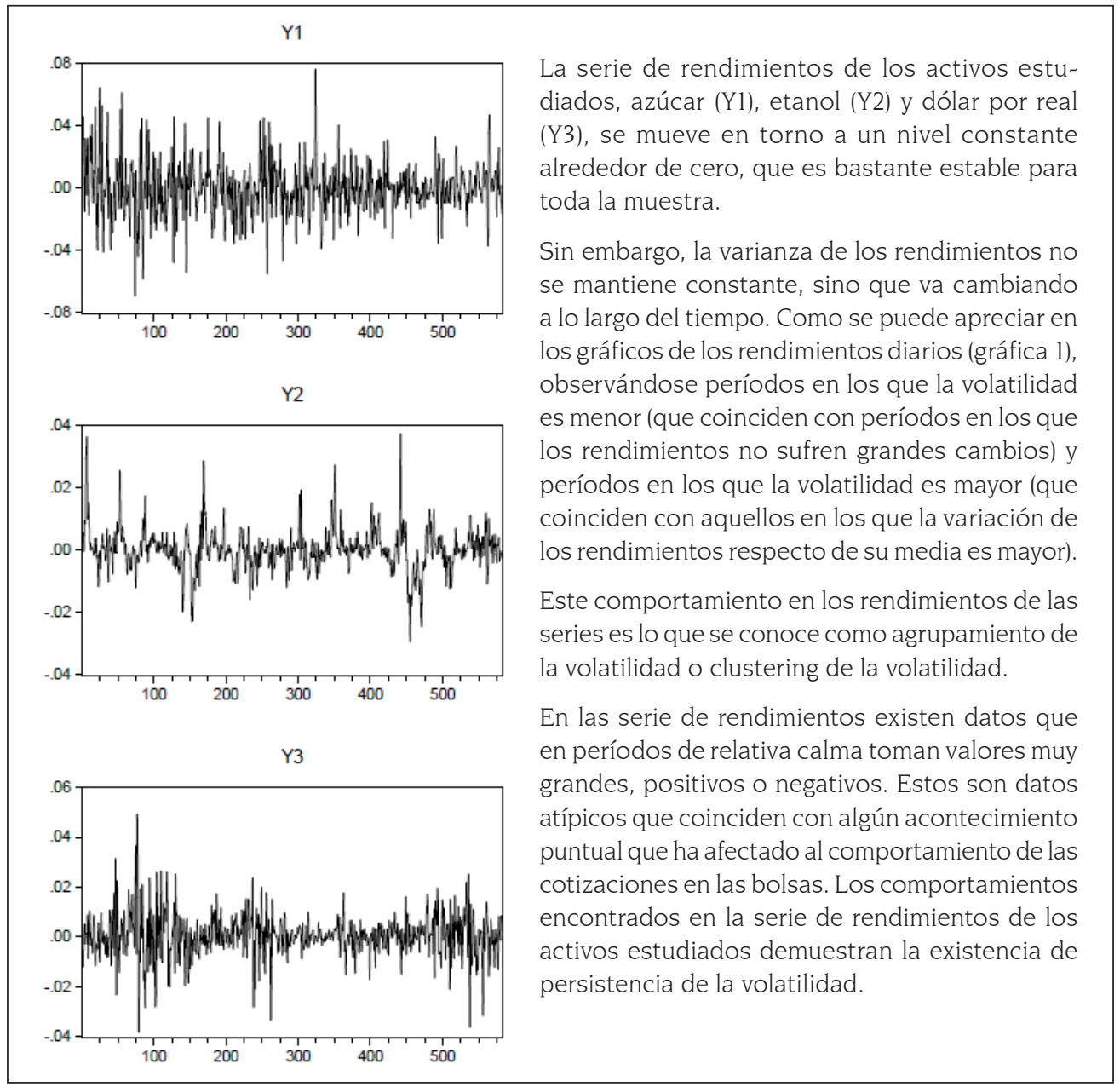

Fuente: elaboración propia 
Gráfica 2. Prueba de normalidad y efecto leverage

\begin{tabular}{|c|c|c|c|}
\hline \multirow[t]{2}{*}{$\begin{array}{l}\text { Date: } 11 / 12 / 13 \\
\text { Sample: } 1582\end{array}$} & \multicolumn{3}{|l|}{ Time: $12: 39$} \\
\hline & $\mathrm{Y} 1$ & Y2 & Y3 \\
\hline Mean & -0.000291 & $8.07 E-05$ & 0.000557 \\
\hline Median & -0.000857 & 0.000000 & 0.000674 \\
\hline Maximum & 0.076185 & 0.037257 & 0.049230 \\
\hline Minimum & -0.069477 & -0.029431 & -0.038300 \\
\hline Std. Dev. & 0.017801 & 0.006798 & 0.009078 \\
\hline Skewness & 0.295716 & 0.510041 & 0.030261 \\
\hline Kurtosis & 4.901716 & 8.411156 & 6.528114 \\
\hline Jarque-Bera & 96.01785 & 734.0251 & 301.4240 \\
\hline Probability & 0.000000 & 0.000000 & 0.000000 \\
\hline Sum & -0.168877 & 0.046865 & 0.323584 \\
\hline Sum Sq. Dev. & 0.183797 & 0.026801 & 0.047799 \\
\hline Observations & 581 & 581 & 581 \\
\hline
\end{tabular}

Y1

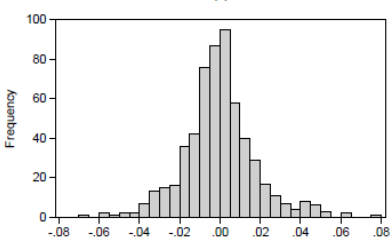

Y2

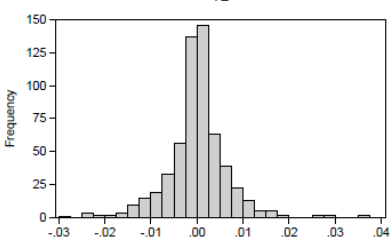

Y3

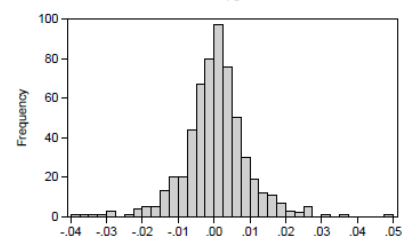

En la gráfica 2 se puede observar que en el centro la densidad de probabilidades es menor en relación con lo normal, en tanto que en los extremos (colas) de la distribución, sobre todo a la izquierda, se concentran una densidad de probabilidades mayor en relación también a lo normal. La ausencia de normalidad se puede demostrar a través de los siguientes estadísticos, para las series de rendimientos del azúcar (Y1), el etanol (Y2) y Dólar por real (Y3):

$\left.1^{\circ}\right)$ la Curtosis es de 4.9 (Y1), 8.4 (Y2) y 6.5 (Y3), muy superior a la que corresponde a una distribución normal estándar de 3.

$2^{\circ}$ ) el valor de la Asimetría (Skewness) es de 0.296 (Y1), 0.51 (Y2) y 0.03 (Y3), diferentes al correspondiente al valor de la distribución normal estándar de 0 .

$\left.3^{\circ}\right)$ el estadístico Jarque-Bera alcanza un valor de 96, 734 y 301, muy alto, ocasionando el rechazo en todos los casos de la hipótesis de normalidad.

En síntesis, los retornos en cuestión muestran un alto valor en la curtosis, excediendo el valor de 3, lo que sugiere que los retornos poseen leptocurtosis comparado con una distribución normal; esto significa una mayor altura que la distribución normal con las colas más anchas. Validándose con esto, las distribuciones de colas pesadas interpretan mucho mejor el comportamiento de estas series de tiempo.

Las series de rendimientos no son simétricas, lo que implica que la respuesta de la volatilidad ante choques de diferentes signos sea asimétrica. Éste hecho es conocido en la literatura econométrica como efecto leverage.

Fuente: elaboración propia

Las series de rendimiento del azúcar, el etanol y el tipo de cambio (Dólar por real), presentan las particularidades que autores como Bollerslev, Engle y Nelson (1994), demostraron empíricamente como representativas de las series financieras, como son: asimétricas y leptocúrticas; presentan ausencia o escasa correlación en las series de rendimientos; poseen varianza cambiante a lo largo del tiempo, alternando períodos de poca volatilidad seguidos de otros de alta volatilidad (agrupamiento de la volatilidad); y existe correlación en los cuadrados de los rendimientos de la serie y estas decrecen de forma lenta hacia cero (persistencia de la volatilidad). 
De acuerdo con lo identificado, se puede afirmar que son aplicables a este caso los modelos de la familia GARCH, ya que las características mencionadas quedan recogidas en estos modelos.

Gráfica 3. QQ - Plot

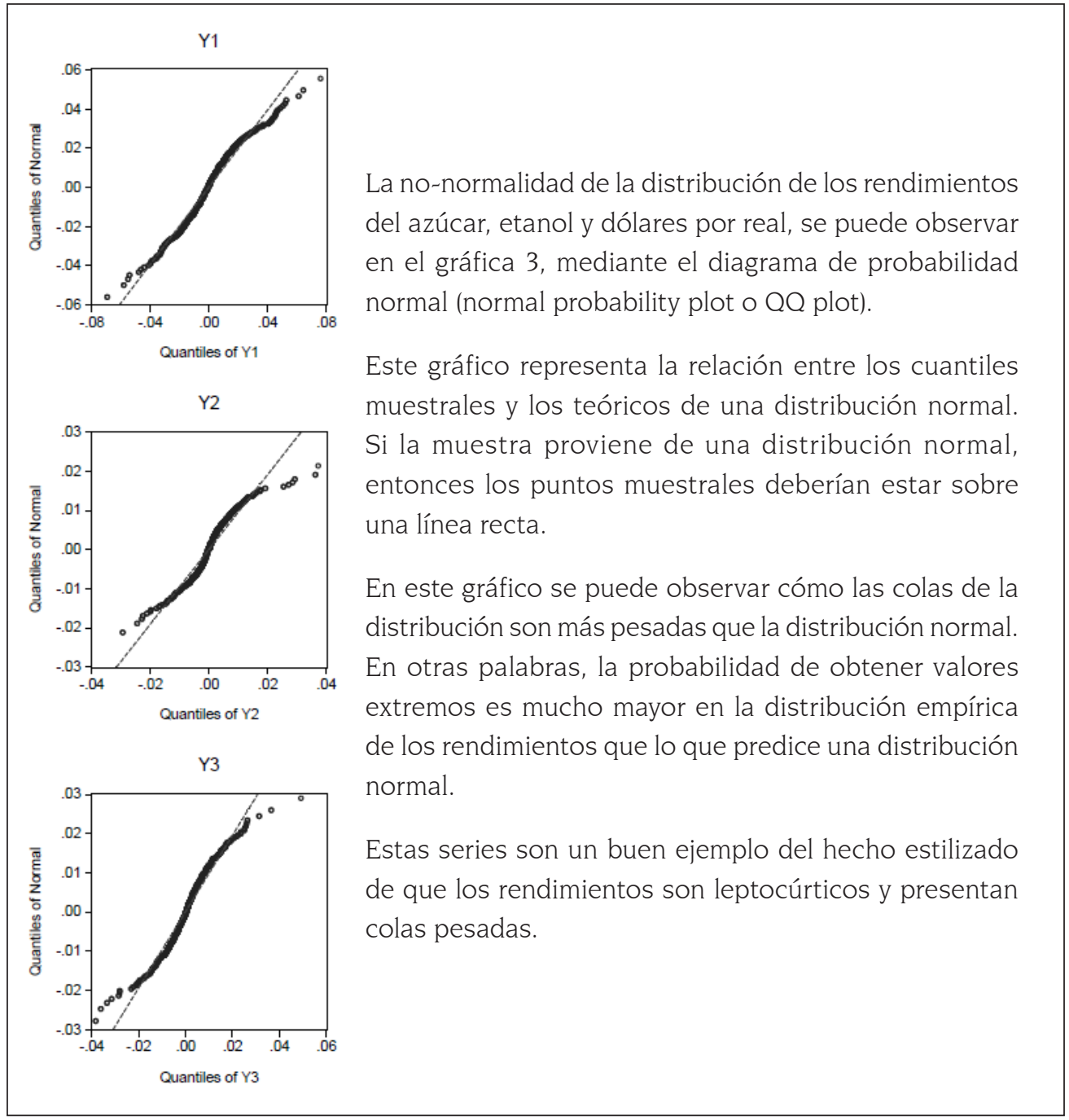

Fuente: elaboración propia

Para interpretar mejor estas series de rendimientos, que son leptocúrticas y presentan colas pesadas, se ha adoptado en este estudio, como mejor aproximación, la distribución t-student, que tiene las colas más anchas para la estimación de los modelos VECH Diagonal y BEKK Diagonal, en especial porque los valores extremos son los más importantes para los actores de los mercados financieros y 
Arody Ortiz Alvarado - Luis Eduardo Girón

entidades reguladoras.

Estimación de modelos GARCH Multivariados

Tabla 2. Parámetros estimados de los modelos GARCH multivariados

\begin{tabular}{|c|c|c|c|c|c|c|}
\hline \multicolumn{7}{|c|}{ Panel A. Modelos estimados } \\
\hline \multicolumn{4}{|c|}{ Modelo BEKK diagonal } & \multicolumn{3}{|c|}{ Modelo VECH diagonal } \\
\hline Parámetros & Valor & Errores & Valor $-p$ & Valor & Errores & Valor- $p$ \\
\hline$C_{i 1}$ & $-5,94 \mathrm{E}-06$ & 0,000681 & 0,9930 & $-0,000136$ & 0,000684 & 0,8424 \\
\hline $\mathrm{C}_{2}$ & 0,000168 & 0,000227 & 0,4609 & $7,89 \mathrm{E}-05$ & 0,000224 & 0,7248 \\
\hline $\mathrm{C}_{3}$ & 0,000115 & 0,000320 & 0,7200 & 0,000295 & 0,000284 & 0,2991 \\
\hline$\omega_{11}$ & $9,53 \mathrm{E}-07$ & $3,05 \mathrm{E}-07$ & 0,0018 & $1,63 \mathrm{E}-06$ & $6,74 \mathrm{E}-07$ & 0,0157 \\
\hline$\omega_{12}$ & $3,33 \mathrm{E}-06$ & $3,69 \mathrm{E}-06$ & 0,3665 & $1,14 \mathrm{E}-06$ & $2,25 E-06$ & 0,6110 \\
\hline$\omega_{13}$ & $-7,93 \mathrm{E}-07$ & $3,97 \mathrm{E}-07$ & 0,0457 & $-1,37 \mathrm{E}-06$ & $5,85 \mathrm{E}-07$ & 0,0192 \\
\hline$\omega_{22}$ & 1,99E-05 & $1,49 \mathrm{E}-06$ & 0,0000 & $1,26 \mathrm{E}-05$ & $1,45 \mathrm{E}-06$ & 0,0000 \\
\hline$\omega_{23}$ & $-4,47 \mathrm{E}-07$ & $1,28 \mathrm{E}-06$ & 0,7277 & $-4,67 \mathrm{E}-07$ & $9,01 \mathrm{E}-07$ & 0,6041 \\
\hline$\omega_{33}$ & $1,31 \mathrm{E}-06$ & $8,42 \mathrm{E}-05$ & 0,1197 & 1,37E-06 & $4,20 \mathrm{E}-07$ & 0,0011 \\
\hline$\alpha_{11}$ & 0,054970 & 0,023628 & 0,0200 & $-0,0011769$ & 0,005270 & 0,0255 \\
\hline$\alpha_{12}$ & \multirow{4}{*}{0,816699} & & & 0,078078 & 0,043671 & 0,0738 \\
\hline$\alpha_{13}$ & & & & 0,013379 & 0,016608 & 0,4205 \\
\hline$\alpha_{22}$ & & 0,046135 & \multirow[t]{2}{*}{0,0000} & 0,590532 & 0,065318 & 0,0000 \\
\hline$\alpha_{23}$ & & & & $-0,069834$ & 0,065667 & 0,2876 \\
\hline$\alpha_{33}$ & 0,320628 & 0,025557 & 0,0000 & 0,139382 & 0,021344 & 0,0000 \\
\hline$\beta_{11}$ & 0,995078 & 0,001475 & 0,0000 & 0,979274 & 0,006428 & 0,0000 \\
\hline$\beta_{12}$ & & & & 0,484232 & 0,051965 & 0,0000 \\
\hline$\beta_{13}$ & & & & 0,914072 & 0,010822 & 0,0000 \\
\hline$\beta_{22}$ & 0,182339 & 0,077929 & 0,0193 & 0,239443 & 0,051319 & 0,0000 \\
\hline$\beta_{23}$ & & & & 0,451991 & 0,048907 & 0,0000 \\
\hline$\beta_{33}$ & 0,943104 & 0,008766 & 0,0000 & 0,853211 & 0,019375 & 0,0000 \\
\hline & & & Theoss & isticas & & \\
\hline & Modelo BEKK & K diagonal & & Modelc & $\mathrm{ECH}$ diagona & \\
\hline Prue & & Valor & & Prueba & & \\
\hline $\begin{array}{l}\text { Akaike } \\
\text { Schwarz } \\
\text { Estacionari } \\
\left(\alpha_{i i}^{2}+\beta_{i i}^{2}\right)<\end{array}$ & & $\begin{array}{l}-19.56 \\
-19.45 \\
0.993,0.7 \text { y } 0.9\end{array}$ & & $\begin{array}{l}\text { e } \\
\text { ionariedad } \\
\left.\beta_{i i}\right)<1\end{array}$ & $\begin{array}{l}-19.62 \\
-19.48 \\
0.991,0 .\end{array}$ & 0.993 \\
\hline
\end{tabular}

Fuente: elaboración propia. 
Las ecuaciones de la media y la covarianza condicional son estimadas en forma conjunta a través del método de máxima verosimilitud usando la rentabilidad diaria de las series financieras de SB1 (Azúcar), BAAWHYDP (Etanol) y USDBRL (Dólar por real). Para facilitar el análisis de los resultados, las series financieras son representadas por los índices yl (Azúcar), y2 (Etanol) y y3 (Dólar por real). La tabla 2 proporciona los resultados de los parámetros estimados de los modelos seleccionados con innovaciones T-student durante el período de estudio.

Los modelos GARCH trivariados convergen después de 31 iteraciones para el modelo Diagonal VEC, y 14 iteraciones para el modelo Diagonal BEKK. Las relaciones de los rendimientos son capturadas por los parámetros estimados $C_{1}, C_{2} y C_{3}$ en los dos modelos. En los dos casos, los parámetros $C_{1}, C_{2} y C_{3}$ no son significativos estadísticamente, para un nivel de significancia del $1 \%$ y $5 \%$, lo cual indica que la media no condicionada de los rendimientos de los precios de las tres series (yl, y2, y y3) es igual a cero en el largo plazo, es decir, no hay predictibilidad de los retornos de los precios ${ }^{4}$.

Analizando los resultados de las ecuaciones de la covarianza condicional, se puede observar que los parámetros estimados de la diagonal de la matriz C son todos significativos, excepto el parámetro $\omega_{12}$ y $\omega_{23}$ para el modelo VEC diagonal y los parámetros $\omega_{12}, \omega_{23}$ y $\omega_{33}$ para el modelo BEKK. Por su parte, todos los elementos de la diagonal de las matrices A y B, que capturan los efectos ARCH y GARCH, son en gran medida significativos para los dos modelos GARCH multivariados. Este hecho sustenta que los efectos ARCH y GARCH son esenciales en ambas series financieras.

Los elementos fuera de la diagonal de las matrices A y B capturan los efectos colaterales de mercado, también conocidos como choques spillover y volatilidad spillover, entre las dos series financieras.

Las versiones no permiten la dependencia dinámica entre las series de volatilidad.

$4 \quad$ El retorno de los precios es igual a $\ln \left(\frac{\mathrm{P}_{t}}{\mathrm{P}_{t-1}}\right)=c+\varepsilon$. 


\section{Gráfica 4. Correlaciones condicionales - Modelo Diagonal BEKK}

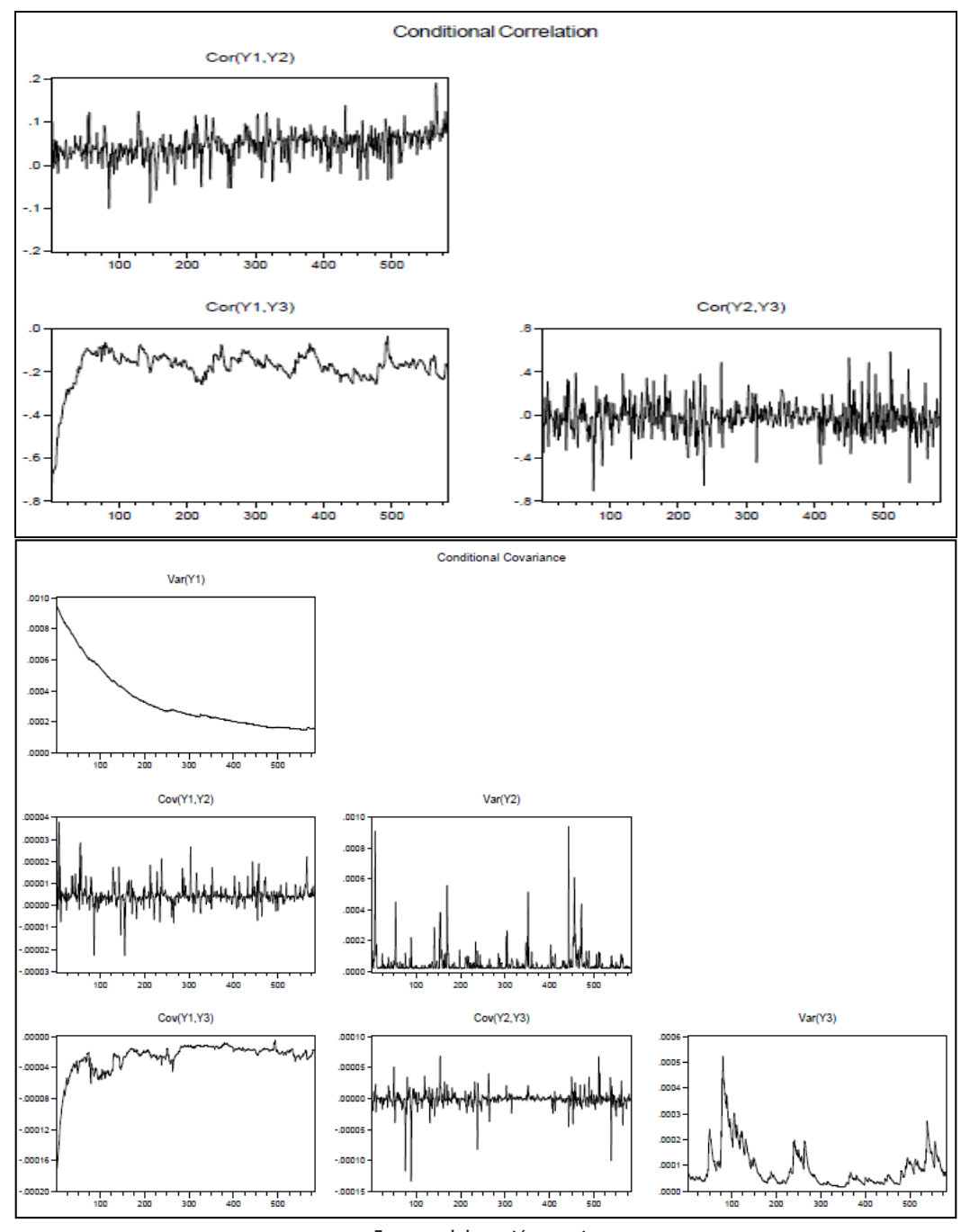

Fuente: elaboración propia

La leve correlación que se evidencia entre la volatilidad de los rendimientos del azúcar y la volatilidad de los rendimientos del etanol, en el modelo BEKK, son en gran parte explicados por la intervención del estado Brasileño en los precios del etanol a través del subsidio que existe al consumo de la gasolina. Así, el precio del etanol en Brasil no recibe en su totalidad el choque de los movimientos de los commodities energéticos (caso del azúcar) como consecuencia de aspectos fundamentales y especulativos que operan a nivel global. 
En los momentos de baja volatilidad se aprecia una leve correlación y en los momentos de alta volatilidad se aprecia una semifuerte correlación. Esto puede encontrar explicación en que el comportamiento del precio del azúcar posee otros determinantes fundamentales fuertes en el mercado, como son: el stock de inventarios en el mundo como consecuencia de la producción en los diferentes países, el clima que repercute en los rendimientos agrícolas y fabriles, y la participación en las Bolsas de los fondos de especulación que utilizan los commodities como inversión.

Asimismo, la leve correlación, siempre negativa, que se observa entre la volatilidad de los rendimientos del azúcar y la volatilidad de los rendimientos del tipo de cambio (Dólar por real), a excepción del primer semestre del año 2011 donde se presentó una alta correlación de -0.8 que coincide con la época en que el Banco Central de Brasil empezó a devaluar el real con mayor fuerza, en el modelo BEKK es explicada por la intervención del Banco Central Brasileño sobre la moneda, disminuyendo en alguna medida el choque que se da en la volatilidad de la rentabilidad del azúcar por hechos fundamentales que ocurren en el ámbito mundial.

La semifuerte correlación que se encuentra entre la volatilidad de los rendimientos del etanol y la volatilidad de los rendimientos del tipo de cambio (Dólar por real), en el modelo BEKK, es explicada por la intervención del Estado en el precio del combustible y la intervención que realiza el Emisor sobre la moneda (Real). Por lo tanto, se concluye que es posible utilizar la información contenida en el tipo de cambio (Dólar por real) para proyectar la volatilidad de los rendimientos del etanol que se produce y comercializa en Brasil.

Otro hallazgo derivado de los resultados de los parámetros estimados es que el modelo VECH y el modelo BEKK diagonal son estacionarios. La estacionariedad en los modelos VECH diagonal y BEKK diagonal es alcanzada cuando las sumas $\alpha_{\mathrm{ii}}+\beta_{\mathrm{ii}}$ y $\alpha_{\mathrm{ii}}^{2}+\beta_{\mathrm{ii}}^{2}$ son menores a 1, es decir, 0.991, 0.83, 0.993 y $0.993,0.7$ y 0.992 , respectivamente. Un aspecto clave para la selección del modelo óptimo es comparar el valor de los criterios de información de Akaike (AIC) y Schwarz (BIC). En términos de ajuste, el modelo VECH diagonal es similar al modelo BEKK diagonal, debido a que presenta valores Akaike y Schwarz muy cercanos (-19.62 contra -19.56, y -19.48 contra -19.45). Sin embargo, el modelo VECH Diagonal es menos parsimonioso al tener más parámetros y, tal como se mencionó antes de la estimación, bajo esta representación no se puede garantizar que la matriz de covarianzas $H_{t}$ sea definida positiva, y en efecto la matriz A resultó indefinida. Por lo tanto, el modelo que presenta mayor robustez es el modelo BEKK.

Tal como lo han manifestado muchos autores, la elección de una forma cuadrática para los errores de la varianza condicional ocasiona que el impacto de los valores 
pasados sobre la volatilidad actual sea tan sólo función de dicha magnitud pasada, lo cual podría resultar impreciso en la dinámica de las series financieras, en las que la volatilidad tiende a ser más alta cuando se presenta una caída, en comparación a cuando se presenta un alza.

Pronóstico de la volatilidad del modelo GARCH-MULTIVARIADO BEKK

- Pronóstico a partir de la estimación del modelo BEKK diagonal con los rendimientos de los precios spot del azúcar, etanol y USDBRL

Tabla 3. Resultado pronóstico volatilidad retornos spot azúcar - BEKK

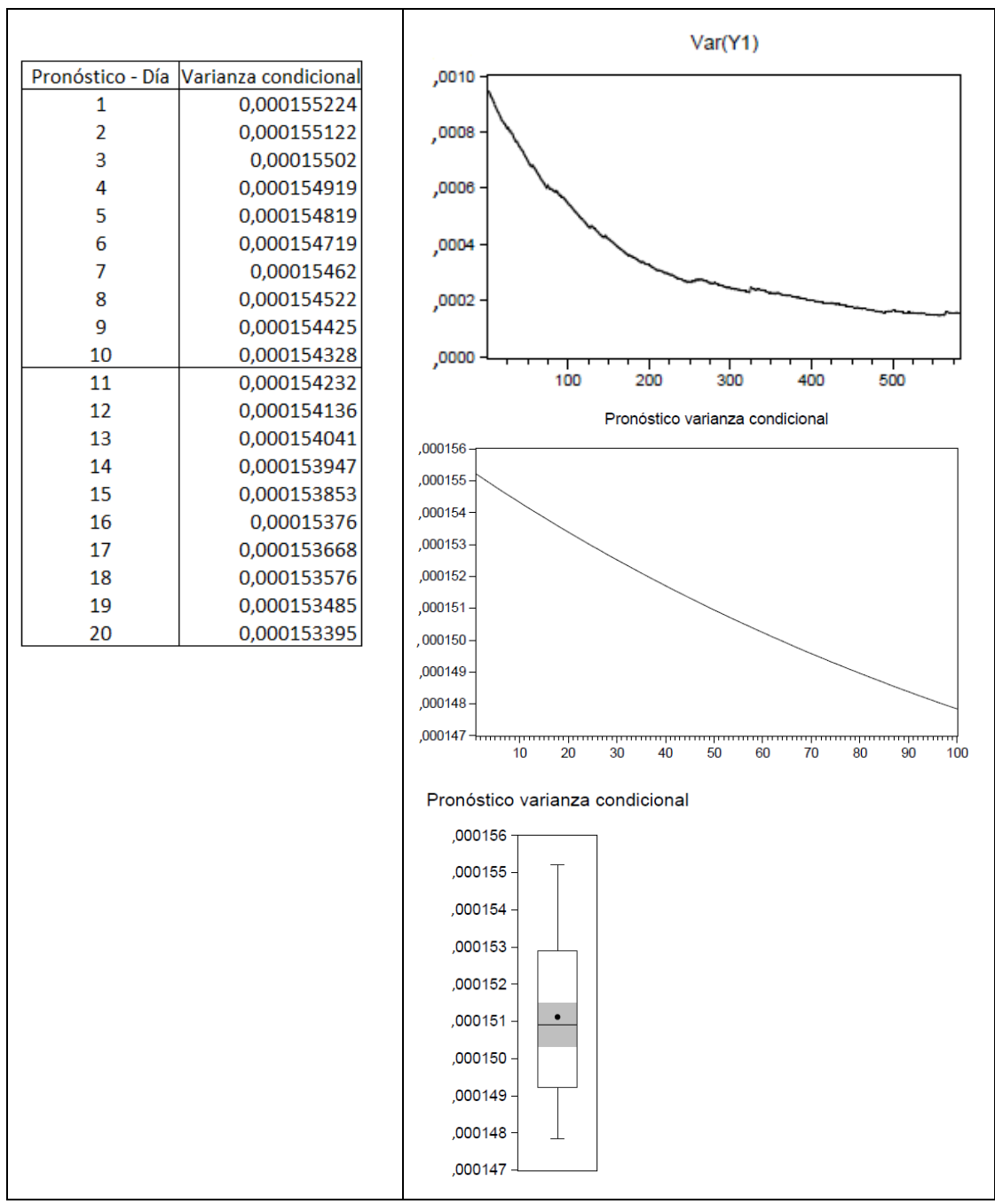

Fuente: elaboración propia 
Predicción de volatilidad de la rentabilidad diaria del mercado del azúcar y su aplicación en la razón de cobertura

Tabla 4. Resultado pronóstico volatilidad retornos futuros azúcar - BEKK

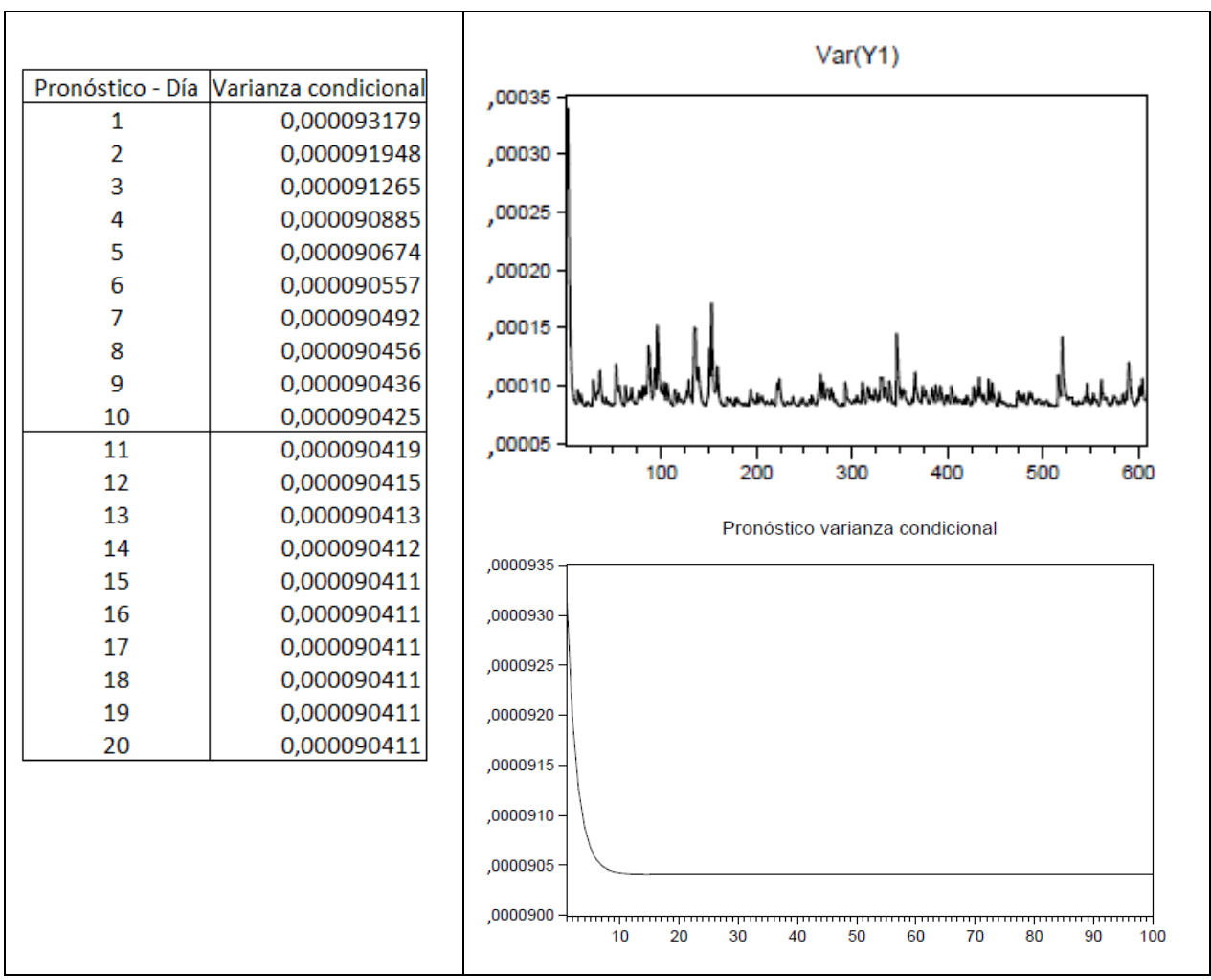

Fuente: elaboración propia

Estimación de la razón de cobertura de mínima varianza

Utilizando los pronósticos de las varianzas de los rendimientos de los precios de contado y futuro del azúcar obtenidas con el modelo trivariado BEKK, con las series de rendimientos de los precios de contado y futuro del azúcar, etanol y Dólar por real, y utilizando la correlación promedio obtenida con el modelo bivariado BEKK, con las series de rendimientos de los precios de contado y futuro del azúcar, se calculará la razón de cobertura para 1, 10 y 20 días:

Como se explicó con anterioridad, el índice de cobertura óptima es invariante en el tiempo, y se calcula a partir de datos históricos. Sin embargo, las desviaciones estándar y la correlación entre los retornos de los precios contado y futuro están cambiando en el tiempo, y podrían ser pronosticadas a partir de un modelo GARCH multivariante, con la siguiente fórmula:

$$
h_{t}=\rho_{t} \frac{\sigma_{s, t}}{\sigma_{F, t}}
$$


Donde $h_{t}$ es razón de cobertura cambiante en el tiempo. $\sigma_{\mathrm{s}, \mathrm{t}}$ es la desviación estándar del cambio en el precio spot durante la cobertura en el momento t, $\sigma_{\mathrm{F}, \mathrm{t}}$ es la desviación estándar del cambio en el precio forward durante la cobertura en el momento t, y $\rho_{\mathrm{t}}$ es la correlación, durante la cobertura, entre el cambio en el precio spot y el cambio en el precio forward en el momento $t$.

Gráfica 5. Media del coeficiente de correlación rentabilidad mercado azúcar según precio de contado y futuro

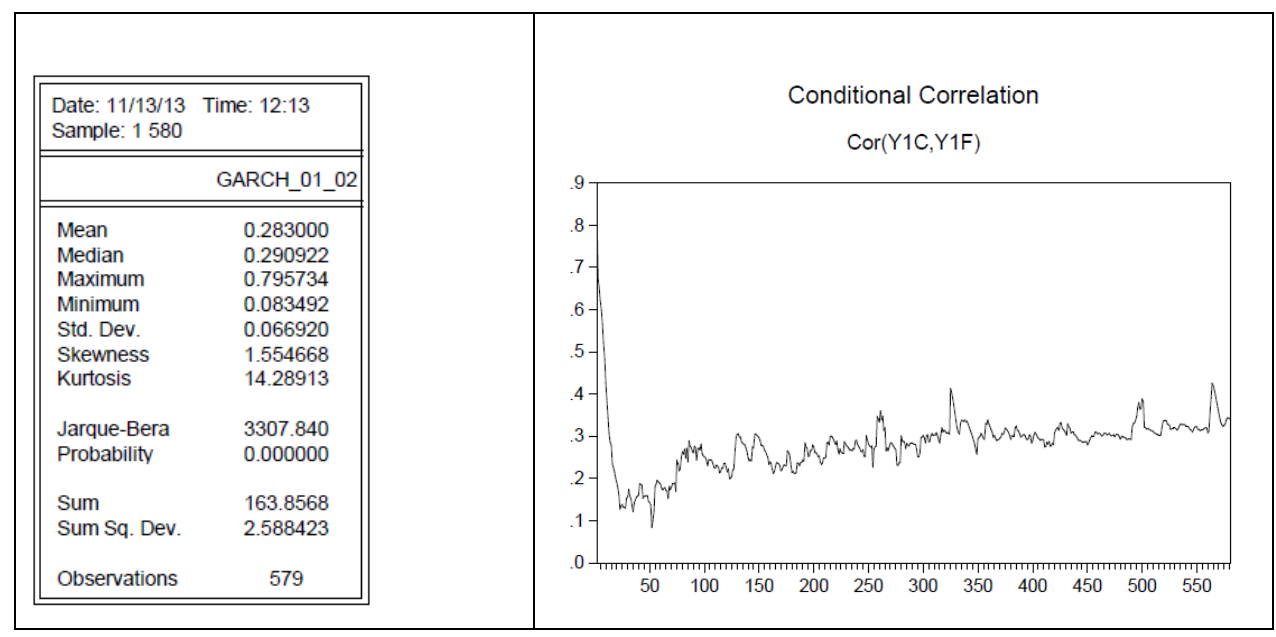

Fuente: elaboración propia

Tabla 5. Resultado razón de cobertura

\begin{tabular}{|c|c|c|c|c|c|}
\hline & & & & & Razón de cobertura \\
\hline $\begin{array}{c}\text { Período } \\
\text { (días) }\end{array}$ & $\begin{array}{c}\text { Razón de } \\
\text { cobertura }\end{array}$ & $\begin{array}{c}\text { Correlación } \\
\text { media }\end{array}$ & $\begin{array}{c}\text { Desviación } \\
\text { rendimiento } \\
\text { precio } \\
\text { contado } \\
\end{array}$ & $\begin{array}{c}\text { Desviación } \\
\text { rendimiento } \\
\text { precio futuro }\end{array}$ & $\begin{array}{l}.367 \\
.366 \\
.365\end{array}$ \\
\hline$t$ & 0.37 & 0.283 & 0.01245889 & 0.009652927 & $364-$ \\
\hline$t+1$ & 0.37 & 0.283 & 0.0124548 & 0.009588952 & .363 \\
\hline$t+10$ & 0.37 & 0.283 & 0.01242288 & 0.009509206 & $362-$ \\
\hline$t+20$ & 0.37 & 0.283 & 0.01238527 & 0.00950847 & $10 \quad 20 \quad 30$ \\
\hline $\mathrm{t}+100$ & 0.36 & 0.283 & 0.01215887 & 0.00950847 & \\
\hline
\end{tabular}

Fuente: elaboración propia

De acuerdo con los resultados de la razón de cobertura, a corto plazo, la razón de cobertura es 0.37 .

Como cada contrato sobre azúcar en NYBOT se negocia sobre 50.84 toneladas de azúcar. Por lo tanto, si el productor espera vender 25 contratos de azúcar, el 
número de contratos de futuro óptimo tomando cobertura en la posición de mayo de 2014 que se negocia en NYBOT, de acuerdo con la razón de cobertura óptima, será aproximadamente de 9; dependiendo del día que realice la compra del contrato de futuro $(t, t+1, t+10, o t+20)$.

\section{CONCLUSIONES Y RECOMENDACIONES}

Al analizar los mercados del azúcar, el etanol y el tipo de cambio (Dólar por real) en forma simultánea, bajo el argumento de que estos tres mercados han sido, a través de la historia, vinculados entre sí, se logró validar que están correlacionados; por momentos, de manera semifuerte, y en otros momentos, en forma débil. Y esto resulta mucho mejor que no conocer nada acerca de los enlaces de la volatilidad de los rendimientos o acerca de los factores aleatorios que modifican su evolución. Por lo tanto, la predicción de las volatilidades y correlaciones es la base para estructurar cualquier estrategia de cobertura de precios.

De acuerdo con el comportamiento de la serie de volatilidad de los rendimientos del azúcar, y su tendencia, se podría afirmar que durante el periodo comprendido entre agosto 24 de 2013 y octubre 24 de 2013 (últimos 60 días) la volatilidad se encuentra en una zona de calma, es decir una zona en la que los rendimientos de los precios no han experimentado fluctuaciones fuertes.

Los pronósticos resultantes de la volatilidad condicional de los retornos de los precios del azúcar muestran la rapidez con la que tienden a su media incondicional, lo cual indica que el horizonte del pronóstico de estos modelos es muy corto, proporcionando predicciones inmediatas.

Los resultados de la correlación media, entre la rentabilidad del mercado del azúcar de acuerdo con el precio de contado, y la rentabilidad del mercado del azúcar de acuerdo con el precio futuro, reflejan que durante el mes de octubre/13 el coeficiente de correlación ha estado alejado de la media, ubicándose cerca de 0.4. Esto podría sugerir que para el cálculo de la razón de cobertura de muy corto plazo, por su alta sensibilidad al coeficiente de correlación, valdría la pena utilizar el coeficiente de correlación del mes de octubre de 2013 y estar comparando su resultado con el obtenido al utilizar el coeficiente de correlación media y contrastar con el que se obtenga cuando se conozca la información real.

Las correlaciones y su análisis son insumos críticos para el entendimiento de la volatilidad de los rendimientos de los activos y para su predicción. Por lo tanto, para estructurar coberturas se requieren estimaciones de la correlación entre los rendimientos de los activos de interés; si las correlaciones y las volatilidades están 
cambiando, entonces la razón de cobertura debe ser ajustada siempre para tener en cuenta la información más reciente.

En la razón de cobertura cambiante en el tiempo, los parámetros $\sigma_{\mathrm{s}, \mathrm{t}}$ (desviación estándar del cambio en el precio spot), $\sigma_{\mathrm{F}, \mathrm{t}}$ (desviación estándar del cambio en el precio futuro), y $\rho_{\mathrm{t}}$ (coeficiente de correlación), se estiman mediante datos históricos. Esto supone que el futuro, en cierta medida, será como el pasado.

El beneficio para el productor averso al riesgo al realizar la cobertura utilizando la razón de varianza mínima es el porcentaje de la varianza que logra eliminar.

En general en los modelos GARCH, cuando se utiliza los datos históricos para la estimación de la volatilidad condicional y su predicción, se le está dando un valor importante a la tendencia del pasado. Por lo tanto, se observa un punto de encuentro "similitud" con la teoría chartista (análisis técnico), que se caracteriza por predecir el comportamiento futuro de los precios a partir de los patrones observados en el pasado.

Como se mencionó, los elementos fuera de la diagonal de las matrices A y B capturan los efectos colaterales de mercado, también conocidos como choques spillover y volatilidad spillover, entre las dos series financieras, y dado que los modelos estimados, en esta investigación fueron las versiones VECH Diagonal y BEKK Diagonal que no permiten la dependencia dinámica entre las series de volatilidad, se recomienda explorar otros software para estimar las versiones completas de los modelos VECH y BEKK.

El mercado financiero en general está afectado por imperfecciones y distorsiones, que lo hacen incierto, y transcurridos cinco años desde el inicio de la crisis financiera global se podría afirmar que la forma en que se comporta el mercado financiero internacional sigue siendo la misma. En este sentido, es importante señalar que los productores de azúcar en países emergentes tienen a su alcance las mismas herramientas de gestión de riesgo, como la expuesta en esta investigación, que otras firmas en el resto del mundo, ya sea un país desarrollado o una economía emergente (independiente del contexto de los negocios). Por lo tanto, el uso de estas herramientas se constituirá en un factor de diferenciación, cuando las firmas incorporen en su estrategia de negocios la capacidad de cuestionar con frecuencia la manera en que se gestiona el riesgo y desafiar la validación empírica de ciertos supuestos.

Sería de mucha utilidad analizar en trabajos futuros, cuando la gasolina deje de ser subsidiada en Brasil, la correlación de la volatilidad de los rendimientos del azúcar con la volatilidad de los rendimientos del etanol y la volatilidad de los 
rendimientos del tipo de cambio en Brasil (Dólar por real); lo que esto significa, desde el punto de vista intuitivo, es que la predicción de la volatilidad para la estructuración de coberturas sobre commodities tiene un potencial alto a través de los modelos multivariados GARCH, en la medida de ausencia de restricciones a la formación de los precios en el mercado, de tal forma que pueda darse de manera eficiente.

Se recomienda para futuros estudios estimar la volatilidad para las demás posiciones de futuros del azúcar que se cotizan en NYBOT (Junio, Agosto, y Octubre) de manera simultánea, utilizando los modelos multivariantes, para construir un portafolio de razones de cobertura.

Por último, una línea de trabajo en que resultaría interesante continuar utilizando la metodología aplicada en esta investigación sería el estudio de la serie de la rentabilidad del mercado del azúcar en Colombia de acuerdo con el precio interno, para establecer si al igual que la serie de la rentabilidad del mercado del azúcar, de acuerdo con el precio internacional, sigue una trayectoria de caminata aleatoria; y establecer el nivel de correlación entre la volatilidad de la rentabilidad del mercado del azúcar en Colombia y la volatilidad de la rentabilidad del mercado internacional del azúcar.

\section{BIBLIOGRAFÍA}

Arias, Joaquín; Chavarría, Hugo; Jara, Byron; Krivonos, Ekaterina; Rodrigues, Mónica; Rodríguez, Adrián; Salcedo, Salomón; Saucedo, Alberto; Sotomayor, Octavio y Trejos, Rafael (2011). Perspectivas de la agricultura y del desarrollo rural en las Américas: una mirada hacia América Latina y el Caribe. Volatilidad de precios en los mercados agrícolas (2000-2010): implicaciones para América Latina y opciones de políticas. En: Boletín CEPAL/FAO/IICA, No.1, p. 1-21.

ASOCAÑA- Asociación de Cultivadores de Caña de Azúcar de Colombia (2013). Aspectos generales del sector azucarero. Cali, Mayo de 2013, 171p. [Consultado 3 de Julio de 2013].

Bollerslev, T. (1986). Generalized autoregresive conditional heteroscedasticity. En: Journal of Econometrics, No. 31, p. 307-327.

Bollerslev, T. Engle R. y Wooldridge J. (1988) A Capital Asset Pricing Model with Time Varying Covariances. En: Journal of Political Economy, Vol. 96, No. 1, p. 116-131.

Bollerslev, Tim (1990). Modeling the Coherence in Short-run Nominal Exchange Rates: A Multivariate Generalized ARCH Model. En: The Review of Economics and Statistics, Vol. 3, No. 72, p. $498-505$. 
Bollerslev, T. Engle R.F. y Nelson D.B. (1994). ARCH models. En: Handbook of Econometrics, Vol. IV. En R.F Engle y D.L. McFadden (eds.), p. 2959-3038.

Brooks, Chris. (2008). Introductory econometrics for finance. New York, Cambridge University Press, $648 \mathrm{p}$.

Diebold Francis X. (2007). Elements of Forecasting. Cincinnati, Thomson South Western, 363 p.

Engle R. (1982). Autoregresive conditional heteroscedasticity with estimates of the variooance of United Kingdom inflation. En: Econométrica, Vol. 50, No. 4, p. 987-1007.

Engle, R. y Kroner, K. (1995). Multivariate simultaneous generalized ARCH, En: Econometric Theory, No 11, p.122-150

Gilbert, Christopher L. (2010). How to Understand High Food Prices. En: Journal of Agricultural Economics, No. 61, p. 398-425.

Hull, John C. (2009). Option, futures and other derivatives. New Jersey, Pearson Prentice Hall, 814 p.

FAO- Organización para la Alimentación y la Agricultura (2012). Volatilidad en los precios de los alimentos y el papel de la especulación. Roma, Julio de 2012, 144p. [Consultado 29 de Julio de 2013].

Riaño, Juanita (1997). El mercado de futuros y la volatilidad del precio internacional del café. En: Ensayos sobre economía cafetera. 11-20, No.13, p. 83-96.

Saban, N.; Cumhur, E. y Ugur S. (2013). Volatility spillover between oil and agricultural commodity markets. En: Energy Economics No 36, p. 658-665.

Serra T. (2011). Volatility spillovers between food and energy markets: A semiparametric approach. En: Energy Economics, No 33, p. 1155-1164.

Tsay Ruey S. (2005). Analysis of Financial Times Series. New Jersey, John Wiley y Sons, 677 p. 